\title{
Role of Tau as a
}

\section{Microtubule-Associated Protein: Structural and Functional Aspects}

\author{
Pascale Barbier ${ }^{1}$, Orgeta Zejneli ${ }^{2,3}$, Marlène Martinho ${ }^{4}$, Alessia Lasorsa ${ }^{2}$, Valérie Belle ${ }^{4}$, \\ Caroline Smet-Nocca ${ }^{2}$, Philipp O. Tsvetkov ${ }^{1}$, François Devred ${ }^{1 *}$ and Isabelle Landrieu ${ }^{2 *}$ \\ ${ }^{1} F a c$ Pharm, Aix Marseille Univ., Centre National de la Recherche Scientifique (CNRS), Inst Neurophysiopathol (INP), \\ Fac Pharm, Marseille, France, ${ }^{2}$ Univ. Lille, Centre National de la Recherche Scientifique (CNRS), UMR 8576, Unité de \\ Glycobiologie Structurale et Fonctionnelle (UGSF), Lille, France, ${ }^{3}$ Univ. Lille, Institut National de la Santé et de la Recherche \\ Médicale (INSERM), CHU-Lille, UMR-S 1172, Centre de Recherche Jean-Pierre AUBERT (JPArc), Lille, France, ${ }^{4}$ Aix Marseille \\ Univ., Centre National de la Recherche Scientifique (CNRS), UMR 7281, Bioénergétique et Ingénierie des Protéines (BIP), \\ Marseille, France
}

\section{OPEN ACCESS}

Edited by:

Jesus Avila,

Autonomous University of Madrid,

Spain

Reviewed by:

Santosh Jadhav,

Institute of Neuroimmunology (SAS),

Slovakia

Cristina Di Primio,

Scuola Normale Superiore di Pisa,

\section{Italy}

*Correspondence:

François Devred

francois.devred@univ-amu.fr Isabelle Landrieu

isabelle.landrieu@univ-lille.fr

Received: 23 May 2019

Accepted: 18 July 2019

Published: 07 August 2019

Citation:

Barbier P, Zejneli O, Martinho M, Lasorsa A, Belle V, Smet-Nocca C, Tsvetkov PO, Devred F and Landrieu I

(2019) Role of Tau as a Microtubule-Associated Protein:

Structural and Functional Aspects. Front. Aging Neurosci. 11:204. doi: 10.3389/fnagi.2019.00204
Microtubules (MTs) play a fundamental role in many vital processes such as cell division and neuronal activity. They are key structural and functional elements in axons, supporting neurite differentiation and growth, as well as transporting motor proteins along the axons, which use MTs as support tracks. Tau is a stabilizing MT associated protein, whose functions are mainly regulated by phosphorylation. A disruption of the MT network, which might be caused by Tau loss of function, is observed in a group of related diseases called tauopathies, which includes Alzheimer's disease (AD). Tau is found hyperphosphorylated in AD, which might account for its loss of MT stabilizing capacity. Since destabilization of MTs after dissociation of Tau could contribute to toxicity in neurodegenerative diseases, a molecular understanding of this interaction and its regulation is essential.

Keywords: post-translational modifications, biophysical methods, Alzheimer's disease, intrinsically disordered proteins, neurodegenerative diseases

\section{INTRODUCTION}

Tau is a microtubule-associated protein (MAP; Weingarten et al., 1975; Witman et al., 1976) that is abundant in the axons of neurons where it stabilizes microtubule (MT) bundles (Binder et al., 1985; Black et al., 1996). Together with other destabilizing MAPs, such as stathmin, Tau plays a central role in MT dynamics by regulating assembly, dynamic behavior and the spatial organization of MTs. Tau and other MAPs that bind to MTs are tightly regulated by a number of factors, including post-translational modifications (PTMs), to ensure the appropriate dynamics of the system (Lindwall and Cole, 1984; Mandelkow et al., 1995; Ramkumar et al., 2018). However, the exact mechanism of assembly and stabilization of MTs by Tau remains challenging to characterize

\footnotetext{
Abbreviations: AD, Alzheimer's Disease; EM, Electron Microscopy; EPR, Electron Paramagnetic Resonance spectroscopy; FRET, Förster Resonance Energy Transfer; FTD, Fronto-Temporal Dementia; GFP, Green Fluorescent Protein; HSQC, Heteronuclear Single Quantum Correlation; IDP, Intrinsically Disordered Protein; MAP, Microtubule Associated Protein; MS, Mass Spectrometry; MT, MicroTubule; NOE, Nuclear Overhauser Effect; NMR, Nuclear Magnetic Resonance spectroscopy; PHF6, Paired Helical Filament Hexapeptide; PRE, Paramagnetic Relaxation Enhancement; PTMs, Post-Translational Modifications; SLD, Stathmin-Like Domain; STD, Saturation Transfer Difference.
} 
due to the inherent dynamics of the system and the disordered nature of Tau. The number of approaches that have been conducted so far to gain knowledge on this particular interaction is striking (Devred et al., 2010; Nouar et al., 2013; Di Maio et al., 2014; Tsvetkov et al., 2019). Tau is probably the most studied MAP because of its implication in a group of neurodegenerative diseases called tauopathies, associated with Tau aggregation into intraneuronal deposits (Brion et al., 1986), such as frontotemporal dementia (FTD), Alzheimer's disease (AD) and progressive supranuclear palsy.

Tau binding to MTs, as well as its propensity to aggregate, are affected by Tau mutations and by Tau PTMs, in particular, its hyper-phosphorylation (Alonso et al., 1994; LeBoeuf et al., 2008). These pathologically modified Tau molecules perturb MT function and axonal transport, contributing to neurodegeneration. Indeed, a reduction of MT density and fast axonal transport is observed in transgenic $\mathrm{AD}$ mouse models that exhibit hyperphosphorylated Tau inclusions in neurons (Cash et al., 2003). Furthermore, Tau mutations carried by FTD patients cause MT-mediated deformation of the nucleus, which in turn results in perturbation of the nucleocytoplasmic transport (Paonessa et al., 2019). In addition, mislocalized Tau in neurons of Tau-overexpressing transgenic mouse brain and of human $\mathrm{AD}$ brain directly interacts with the nucleoporins of the nuclear pore complex. This interaction disrupts the nuclear pore functions of nucleocytoplasmic transport and might contribute to Tau-related neurotoxicity (Eftekharzadeh et al., 2018).

Efforts for clinical intervention in the $\mathrm{AD}$ field have so far mainly focused on blocking the formation of extracellular amyloid $\beta$ deposits, another type of aggregates observed in the brain of $\mathrm{AD}$ patients (Cummings et al., 2017). However, in the last several years, there has been an increase in studies aimed at the therapeutic targeting of Tau. An improved understanding of Tau functions could lead to the development of new strategies of therapeutic interventions (Mudher et al., 2017; Jadhav et al., 2019). This emphasizes the need to understand the finer details of structural and functional aspects of Tau/MTs interaction.

\section{TUBULIN AND MTs}

MTs are hollow cylinders composed of parallel protofilaments of $\alpha$ and $\beta$ tubulin subunits $(\alpha / \beta$ tubulin heterodimer, here named tubulin) assembled head-to-tail, which form a pseudo helical lattice (Amos and Schlieper, 2005; Figure 1). During MT formation, tubulins self-assemble in their guanosine $5^{\prime}$ triphosphate (GTP)-bound state to form a sheet that closes into a $25 \mathrm{~nm}$ outside diameter tube (Chrétien et al., 1995). Whereas the MT core or lattice is constituted of guanosine 5' diphosphate (GDP)-tubulin, a GTP cap forms at MT ends because of the delay between assembly and GTP hydrolysis at the tubulin inter-dimer interface (Carlier et al., 1989; Caplow and Shanks, 1990; Bowne-Anderson et al., 2015; Baas et al., 2016). This cap has been proposed to stabilize growing MTs since its loss induces MT depolymerization, with characteristic curled protofilaments at MT ends (Chrétien et al., 1995). MTs constantly undergo phases of assembly and disassembly in a process called "dynamic instability" (Mitchison and Kirschner, 1984). Due to these intrinsic dynamics of the system, MTs have been difficult to study from a structural point of view. During the past decades, numerous molecular models were used to simulate the mechanism of MT formation, resulting in a more nuanced model than the original GTP-cap one, which now accounts for the diverse regulatory activities of MAPs. In this model named "coupled-random model," GTP hydrolysis occurs with a constant rate for any tubulin in the MT lattice, except for the dimers at the very tip (Bowne-Anderson et al., 2015). GDP-tubulin does not assemble into MTs but forms double rings (Howard and Timasheff, 1986). It was thus originally considered that GTP would allosterically induce a straight conformation of tubulin capable of MT assembly and that GDP would induce a curved conformation favoring disassembly (Melki et al., 1989). It is now generally accepted that GTP-tubulin in solution is curved similarly to GDP-tubulin, based on the MT models derived from electron microscopy (EM) data, up to about $3.5 \AA$ resolution (Zhang R. et al., 2015) and from X-ray crystallography up to $2 \AA$ resolution (Nawrotek et al., 2011; Pecqueur et al., 2012) together with biochemical evidence (Barbier et al., 2010). Tubulin only straightens upon incorporation into MTs (Alushin et al., 2014). In addition to the GDP/GTP dual nature of tubulin, PTMs of tubulin, such as tyrosination and acetylation, modulate MT stability (Baas et al., 2016). In neurons, two MT regions are distinguished (Figure 1): a labile MT region mostly composed of tyrosinated and deacetylated GTP-tubulin, and a stable MT region (or lattice) mostly consisting of assembled detyrosinated and acetylated GDP-tubulin (Baas et al., 2016). These findings together with other recent studies of tubulin modifications-including phosphorylation, polyglycylation, deglutamylation and polyglutamylation-highlight the importance of these PTMs in regulating the different functions of MTs, generating a "tubulin code" (Gadadhar et al., 2017). Indeed, a defect of the deglutamylase CCP1, the enzyme responsible for tubulin deglutamylation, causes infantile-onset neurodegeneration (Shashi et al., 2018), while an excessive polyglutamylation of tubulin reduces the efficiency of neuronal transport in cultured hippocampal neurons (Magiera et al., 2018). Moreover, a default of acetylated tubulin is linked to abnormal axonal transport in several neurodegenerative diseases (Dompierre et al., 2007; Godena et al., 2014; Guo et al., 2017). Altogether, these studies suggest that enzymes responsible for tubulin PTMs could be promising therapeutic targets.

\section{TAU PROTEIN}

There are two major MAPs present in cells from the central nervous system, MAP2 and Tau. The expression of one or the other isoform is regulated during development, and their localizations differ. Tau is mainly found in the axonal compartment, while MAP2 is expressed specifically in cell bodies and dendrites (Melková et al., 2019). Both proteins exist as alternatively spliced isoforms, with some high-molecular weight isoforms for MAP2 (MAP2a and MAP2b). There are six isoforms 


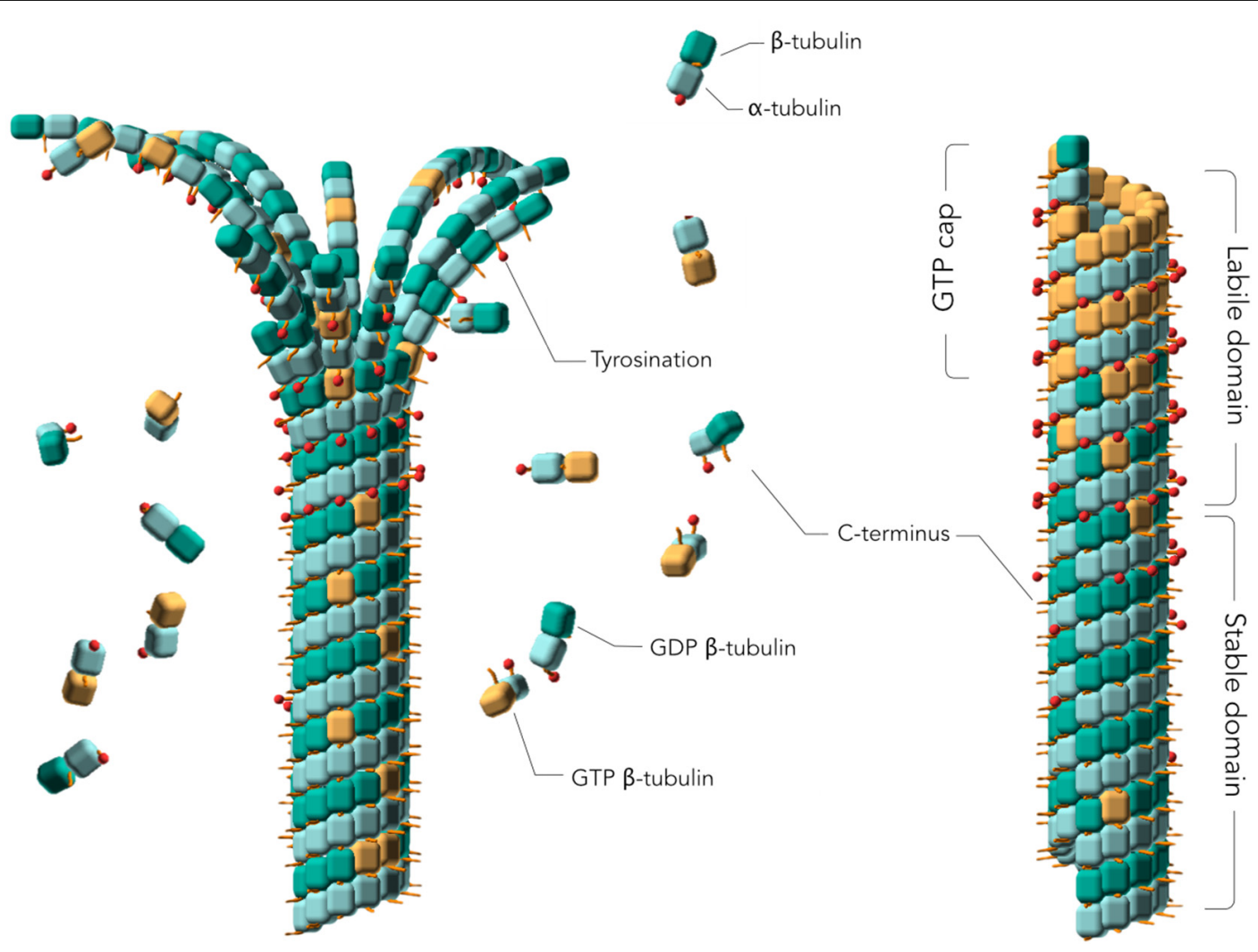

FIGURE 1 | Assembly of tubulins into Microtubules (MTs). Models of a depolymerizing (left) and polymerized (right) MTs. The guanosine $5^{\prime}$ triphosphate (GTP) cap or labile domain is composed mostly of tyrosinated GTP-tubulin. The stable domain is mostly composed of detyrosinated guanosine $5^{\prime}$ diphosphate (GDP)-tubulin. $\beta$-tubulin subunits are represented as orange cubes in their GTP-bound states and green cubes in their GDP-bound states. $\alpha$-tubulin subunits are represented as blue cubes. Red sticks planted on the cubes represent the C-terminal tail of $\alpha$ - and $\beta$-tubulin subunits. Tyrosination is represented by a red dot on the C-terminal tail. Two MT regions are distinguished: a labile MT region mostly composed of tyrosinated GTP-tubulins, including the GTP cap, and a stable MT region mostly consisting of assembled detyrosinated GDP-tubulins. MT depolymerization is characterized by curled protofilaments at MT ends (left).

of Tau protein present in the central nervous system (ranging from 352 to 441 amino acid residues), resulting from mRNA alternative splicing of a single gene (Goedert et al., 1989; Himmler et al., 1989). Tau is divided into functional domains (numbering according to the longest isoform, Figure 2): an $\mathrm{N}$-terminal projection domain Tau(1-165), a proline-rich region Tau(166-242) or PRR, a MT binding region Tau(243-367) or microtubule binding region (MTBR) and a C-terminal domain Tau(368-441). The MTBR consists of four partially repeated sequences R1(243-273), R2(274-304), R3(305-335), and R4(336-367; Figure 2). The isoforms differ by the presence, or not, of one/two insert(s) in the N-terminal domain (N1 and/or $\mathrm{N} 2$ presence or not), and three or four repeat sequences in the MTBR (R2 presence or not). Even though Tau is an intrinsically disordered protein (IDP), it has a tendency to form local secondary structures, in particular, $\beta$-strands in the MTBR and polyproline II helices in the PRR (Mukrasch et al., 2009; Sibille et al., 2012). It has been proposed that Tau N- and C-terminal domains fold back on the central PRR and MTBR domains due to contacts between the $\mathrm{N}$ - and C-termini of the protein (Jeganathan et al., 2006; Mukrasch et al., 2009). This model was initially built using distance measurements based on Förster Resonance Energy Transfer (FRET) detection between pairs of fluorophores attached to Tau (Jeganathan et al., 2006). Moreover, measurements of paramagnetic relaxation enhancement (PRE) by nuclear magnetic resonance spectroscopy (NMR) using paramagnetic centers attached at different positions along the Tau sequence, confirmed proximities of the $\mathrm{N}$ - and C-terminal domains with the central region of Tau (Mukrasch et al., 2009). Several co-factors were reported to enhance Tau structuration including ions such as Zinc (Roman et al., 2019), potentially regulating Tau functions.

\section{MAP FUNCTION OF TAU}

MT dynamics are regulated by proteins that stabilize or destabilize them (van der Vaart et al., 2009). The main role of Tau, one of the stabilizing MAPs, is to protect MTs against depolymerization by decreasing the dissociation of tubulin at both MT ends, resulting in an increased growth rate and decreased catastrophe frequency (Murphy et al., 1977; Trinczek et al., 1995). In vitro, Tau induces MT formation at $37^{\circ} \mathrm{C}$ and tubulin rings at $20^{\circ} \mathrm{C}$ under experimental conditions with no 

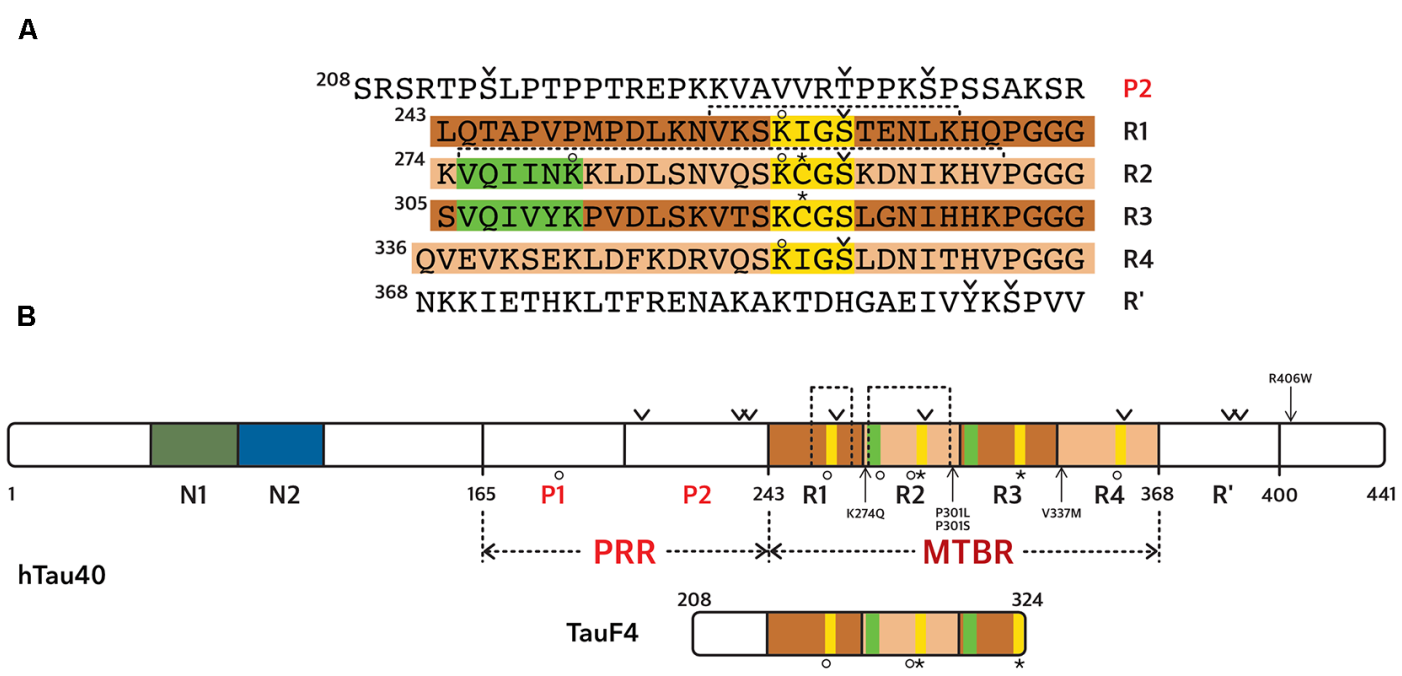

FIGURE 2 | Tau protein sequence and domain organization. The sequence numbering is according to the longest Tau isoform (441 amino acid residues). (A) Amino acid sequence of the microtubule binding region (MTBR) and flanking regions, P2 in the PRR and R' in the C-terminal domain. (B) General scheme of the full-length Tau protein and of TauF4 fragment. (A,B) The MTBR region of Tau consists of four partially repeated sequences, R1 to R4, highlighted in dark and light brown. PHF6* and PHF6 hexapeptides, in R1 and R2 repeats respectively, are highlighted in green and the KXGS motifs in yellow. Phosphorylation sites mentioned in the text are indicated with a v sign, cysteine residues with a star sign and acetylation with a circle. Segments of R1 and R2 shown in Figure $\mathbf{3}$ are indicated with dashed lines.

self-assembly of tubulin alone, suggesting that Tau is a MT inducer in addition to being a MT stabilizer (Weingarten et al., 1975; Devred et al., 2004; Kutter et al., 2016).

Tau was proposed to bind preferentially to the GDP-tubulin from the lattice, in detriment of the GTP-tubulin from the plus-end cap, and in agreement with a stabilizing role of Tau (Duan et al., 2017). However, monitoring of Tau binding to MTs by FRET has shown Tau decoration on MT tips, as well as on the lattice (Breuzard et al., 2013). Furthermore, the capacity of Tau to induce in vitro MT formation is lost when GDP-tubulin is used (Devred et al., 2004). In agreement, Tau depletion in rat cortical neurons results in the loss of MT mass in the axon, predominantly from the labile domain containing tyrosinated tubulin, rather than the stable domain of MTs (Qiang et al., 2018). Green fluorescent protein (GFP)-tagged Tau was indeed observed to be more abundant on the labile domain of the MTs, or GTP-tubulin cap. Based on these observations obtained by quantitative immunofluorescence, the authors propose that Tau is not strictly a MT stabilizer, but rather allows the MTs to conserve long labile domains (Qiang et al., 2018; Baas and Qiang, 2019). However, a more likely explanation is that Tau is necessary to induce tubulin polymerization in the labile region.

Similar to many other IDPs, Tau can undergo phasetransition under in vitro conditions of molecular crowding, resulting in the formation of Tau liquid droplets similar to non-membrane compartments (Hernández-Vega et al., 2017). These Tau-rich drops have the capacity to concentrate tubulin 10 times or higher than the surrounding solution. Nucleation of MTs is observed inside the Tau drops, and MT bundle formation results in the elongation of the drops. This bundle formation could not be reproduced by mimicking the high concentration effect, in the absence of drops. This nucleation environment could support stabilization and provide sufficient plasticity for the formation of the long axonal MT bundles. Although it is not yet known whether this phenomenon is of physiological relevance, GFP-Tau droplets have been observed in transfected neurons (Wegmann et al., 2018).

\section{STRUCTURAL ASPECTS OF TAU MTBR INTERACTION WITH MTS}

Tau structure when bound to MTs has been the object of numerous investigations, leading to several models of Tau/MTs complex. In vitro, single-molecule FRET experiments showed that Tau bound to soluble tubulin adopts an open conformation (Melo et al., 2016), losing the global fold observed for Tau in solution (Jeganathan et al., 2006; Mukrasch et al., 2009; Schwalbe et al., 2014). However, FRET experiments in the cell, using a Tau protein labeled at its $\mathrm{N}$ - and C-termini with fluorophores (Di Primio et al., 2017) show that labeled-Tau bound to MTs displays a global folding, with the $\mathrm{N}$ - and C-termini in close proximity, as described for the "paperclip conformation" of free Tau in in vitro conditions (Jeganathan et al., 2006). This global fold is lost for unbound Tau, detached from the MTs. This discrepancy might be due to the fact that the cytoplasmic environment is much more complex than can be reproduced in in vitro experiments, and Tau might thus bind numerous other cellular partners that could influence its conformation (Di Primio et al., 2017).

Globally, tubulin-bound Tau retains its intrinsically disordered character, and forms a fuzzy complex with tubulin, which might be the reason why Tau binding mode accommodates more than one tubulin (Tompa and Fuxreiter, 2008; Martinho et al., 2018). In agreement, a combination of metal shadowing and cryo-electron microscopy (cryo-EM) 
revealed that Tau is randomly distributed on the MT surface (Santarella et al., 2004). FRET and fluorescence recovery after photo-bleaching in live cells similarly concluded to the distribution of the Tau/MTs interaction along the MTs, characterized by Tau diffusion coupled to binding phases (Breuzard et al., 2013). In-line with these observations, Tau was shown to diffuse along the MTs, bi-directionally and independently from active transport, in a manner described as kiss-and-hop interactions (Hinrichs et al., 2012). These interactions, observed by single molecule tracking in neurons, might explain why Tau does not seem to interfere with motor protein-mediated axonal transport along the MTs (Janning et al., 2014).

Early studies by peptide mapping allowed the first characterization of the interaction as an array of weakly interacting sites, defining the MTBR as the MT binding core, which contains the four highly homologous repeats R1-R4 (Butner and Kirschner, 1991; Goode et al., 2000). Recently, despite the challenges related to the dynamic and fuzzy nature of Tau/MTs interaction described above, the combination of cryo-EM at near-atomic resolution and Rosetta modeling generated models of the interaction. This breakthrough study highlights the crucial residues of Tau MTBR directly mediating the interaction and confirms the longitudinal mode of Tau binding on MTs (Kellogg et al., 2018). The complex used in the study consists of dynamic MTs without stabilizing agents other than Tau. Tau is found along the protofilaments, following the H11-H12 helices that form a ridge at the MT surface and that are close to the point of attachment of the C-terminal tails of tubulin (Figure 3). The observed density spans Tau residues 242-367, covering the MTBR, and is centered on the $\alpha$-tubulin subunit while contacts with $\beta$-tubulin are detected on both sides. To obtain further details, artificial four R1 and four R2 constructs were used and showed that each repeat of the protein adopts an extended conformation that spans both intra- and interdimer interfaces, centered on $\alpha$-tubulin and connecting three tubulin monomers.

A direct interaction of Tau peptides corresponding to residues 245-253 (in R1), 269-284 (in R2), and 300-313 (in R3) is proposed, based on the attenuation of Tau NMR signal upon addition of paclitaxel-stabilized MTs (Kadavath et al., 2015b). The described bound-motif in the R2 repeat closely matches the bound-stretch found in the EM structure of four R2 Tau repeats, but not the bound motif in the $\mathrm{R} 1$, for which the peptide 253-266 is rather proposed as the attachment point for R1 repeat (Kellogg et al., 2018). Additionally, the structure of the bound peptide Tau(267-312; a peptide overlapping R2-R3 repeats) was probed using transfer-NOEs NMR signals. Based on the NOE contacts, which detect spatial proximities, a family of converging conformers were calculated for residues 269-284 (R2) and 300-310 (R3). Both peptides fold into a hairpin-like structure, formed by the conserved PGGG motifs, upon binding to MTs (Kadavath et al., 2015b). However, it should be noted that the electron density in the EM structure models cannot accommodate this hairpin (Kellogg et al., 2018).
The hexapeptides 275-VQIINK-280 in R2 (PHF6* or paired helical filament hexapeptide) and 306-VQIVYK-311 in R3 (PHF6; Figure 2) form an extended structure (Kadavath et al., 2015b), in agreement with the EM observation of an extended conformation for R2 (Kellogg et al., 2018). Both the EM and NMR models are incompatible with previous biochemical results, based on combined fluorescence correlation spectroscopy and acrylodan fluorescence screening, suggesting $\alpha$-helical conformation of the bound-Tau repeats (Li et al., 2015).

The majority of structural data were obtained from the binding of Tau on MTs stabilized by exogenous agents such as Taxol, which facilitates the study by decreasing the dynamics of the system. Still, a number of studies show differences between Tau binding to Taxol pre-stabilized MTs, or to Tau-induced MTs, formed using Tau as an inducer. Kinetics analysis of Tau binding to Taxol-stabilized MTs in comparison with Tau-induced MTs suggests the existence of two different binding sites of Tau to tubulin (Makrides et al., 2004), one overlapping the Taxol binding site localized on $\beta$ tubulin in the inner surface of MTs, as previously suggested (Kar et al., 2003).

Site-directed spin labeling combined with electronparamagnetic resonance spectroscopy (EPR) was used to compare Tau binding mode to Taxol-stabilized MTs and to tubulin when Tau is used as the sole inducer of the polymerization (Martinho et al., 2018). In these experiments, thiol-disulfide exchanges between Tau and tubulin or Taxolstabilized MTs was observed by EPR measurements of paramagnetic labels linked by disulfide bridges to Tau two natural cysteines, or to single-cysteine Tau mutants. Differences in the kinetics of the label release were observed between preformed MTs and Tau-induced MTs, explained by the accessibility of cysteines of tubulin and MTs deduced from available structural data. Localization of the two putative binding sites of Tau on tubulin was proposed (Figure 4). The first one in proximity of Cys 347 of $\alpha$-tubulin subunit could interact with Cys291 of Tau in R2. The second one, located at the interface between two protofilaments, in proximity of Cys131 of $\beta$-tubulin subunit, could interact with Cys 322 of Tau in R3. The first site is in agreement with models proposing that the R2-R3 would encompass the interface between tubulin (Kadavath et al., 2015a; Kellogg et al., 2018). Both sites are less accessible in Taxolstabilized MTs, which might explain the much slower release of the disulfide-linked Tau label when Tau is in interaction with the Taxol-stabilized MTs.

\section{ROLE OF THE REGULATORY REGIONS OUTSIDE OF TAU MTBR}

A number of studies addressed the role of the regulatory regions outside the MTBR. The N-terminal projection domain of Tau modulates the formation of MT bundles (Chen et al., 1992; Rosenberg et al., 2008). This domain, described as a polyelectrolyte polymer brush, is proposed based on atomic force microscopy to exert a repulsive force (Mukhopadhyay and Hoh, 2001). In another model, the electrostatic zipper 


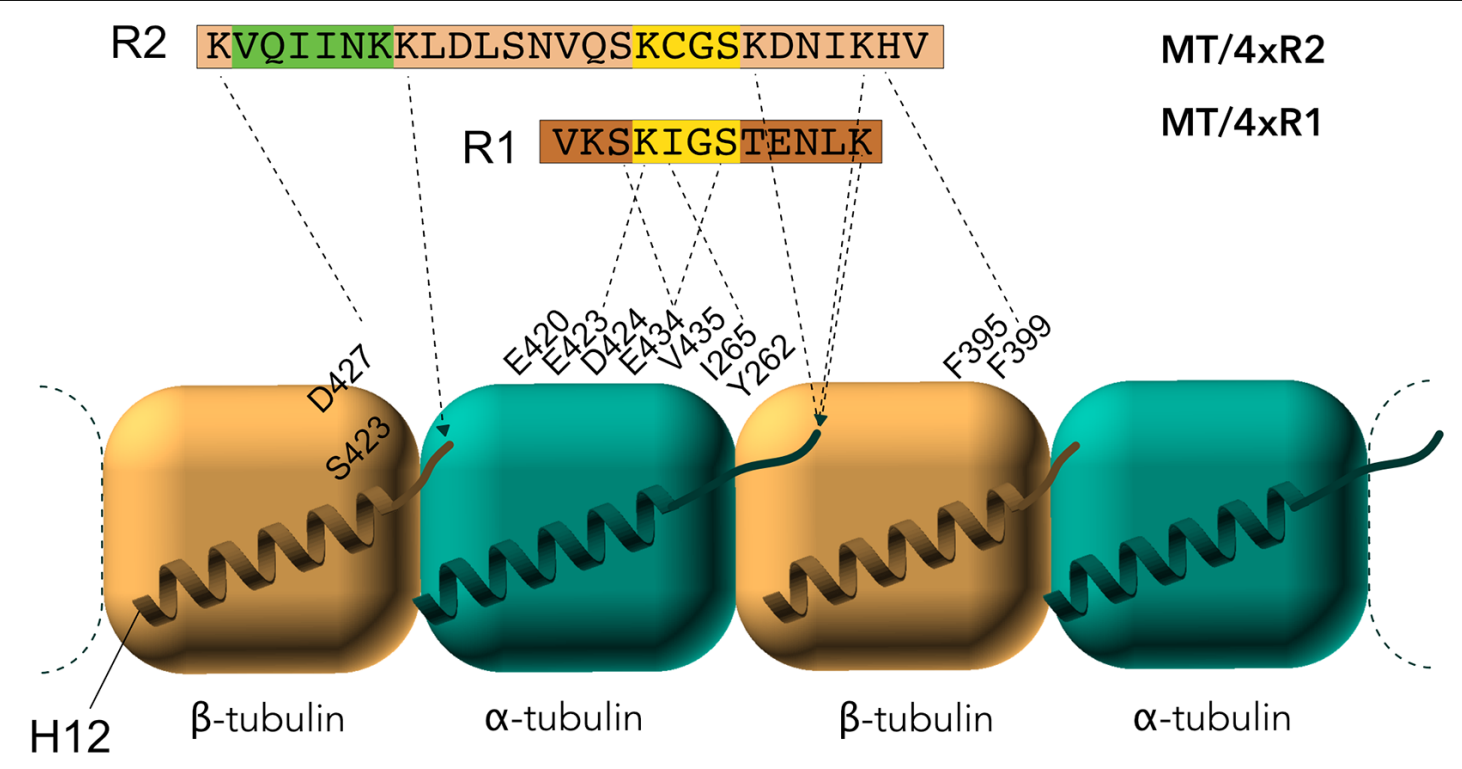

FIGURE 3 | Electron microscopy (EM) models of Tau/MTs interaction. $\alpha$-tubulin subunit is represented as a blue cube and $\beta$-tubulin subunit as a brown cube. H12 $\mathrm{C}$-terminal helix is schematized, the $\mathrm{C}$-terminal tail prolongs the $\mathrm{H} 12$ helix. Model based on cryo-EM coupled to Rosetta modeling: contacts of the R1 repeat with the $\alpha$-tubulin subunit, at the inter-dimer interface: S258 and S262 of R1 make hydrogen bonds with E434; K259 of R1 interacts with an acidic patch formed by E420, E423 and D424; I260 of R1 is in a hydrophobic pocket formed by residues I265, V435 and Y262; K267 of R1 is in contact with the acidic C-terminal tail. Additional contacts for the R2 repeat with $\beta$-tubulin subunit, at the intra-dimer interface: K274 of R2 interacts with an acidic patch formed by D427 and S423; K281 of R2 is in contact with the acidic C-terminal tail of $\beta$-tubulin subunit. The PHF6* peptide (highlighted green) is close to this tail and localize at the intra-dimer interface. Additional contacts for the R2 repeat with $\alpha$-tubulin subunit: K294 and K298 are in contact with the acidic C-terminal tail of $\alpha$-tubulin subunit. Finally, H299 of R2 is buried in a cleft formed by residues F395 and F399 of $\beta$-tubulin subunit.

model, Tau is proposed to organize MT spacing by dimerization of the projection domain and the PRR on adjacent MTs (Rosenberg et al., 2008). Both models might be valid but probably depend on the ionic strength of the solution (Donhauser et al., 2017). The N-terminal domain of Tau remains disordered and highly flexible upon tubulin binding, as seen by both NMR (Kadavath et al., 2015a) and smFRET studies (Melo et al., 2016).

Repeats in the MTBR are required for MT binding and assembly. However, as an isolated fragment, the MTBR is not as efficient in tubulin polymerization and in MT binding as full-length Tau. The regions directly flanking the MTBR, in the PRR and in the C-terminal region, are needed to enhance the binding (Figure 2). Several models have been proposed to describe the roles of MTBR and flanking regions. In the first one, termed the "jaws" model (Gustke et al., 1994; Trinczek et al., 1995; Preuss et al., 1997), the PRR, MTBR, and C-terminal extension domains bind very weakly, if at all, to MTs, and the binding is enhanced when two consecutive domains are associated in a single construct. An NMR study has determined the residues in the PRR and in the downstream repeats that constitute the "jaws" (Mukrasch et al., 2007). The second model proposes that the initial binding of Tau to MTs is mediated by an MTs-binding core within the MTBR, whereas the flanking regions are regulatory (Goode et al., 2000).

The interaction between full-length Tau and Taxol-stabilized MTs allowed mapping of the binding region on the Tau protein at the residue level (Sillen et al., 2007). In particular, both the MTBR and the flanking regions, namely the PRR and 10 amino acid residues located at the C-terminal end of the MTBR, were found to interact with the MTs. In both the PRR and the MTBR, the contribution of basic residues is important for Tau interaction with MTs (Goode et al., 1997; Kadavath et al., 2015b). Additionally, Tau proteolysis products interacting with tubulin were identified by using a complex named $\mathrm{T}_{2} \mathrm{R}$ composed of two tubulins stabilized by the stathmin-like domain (SLD) of RB3 (Gigant et al., 2000; Fauquant et al., 2011). While the isolated fragments from the PRR and MTBR bind weakly to MTs, the Tau(208-324) construct, named TauF4, generated by combining two adjacent fragments included in the PRR and in the MTBR, binds strongly to MTs and stimulates MT assembly very efficiently (Fauquant et al., 2011). The MTBR included in TauF4 (R1, $\mathrm{R} 2$ and the N-terminal part of R3) mostly corresponds to the MT binding core initially proposed by Feinstein and co-workers (Goode et al., 2000). PRE experiments performed by introducing four cysteine mutations on the SLD, located along the protein in proximity of the interface of every tubulin subunit, indicated that TauF4 binds asymmetrically to the two tubulins, with the PRR preferentially located closer to the $\beta$ tubulin subunits (intra-dimer interface). When bound to a single tubulin stabilized by an engineered SLD protein (TR'), a part of the R1 repeat of TauF4 adopts a turn-like conformation, which remains flexible, and thus not in direct contact with $\alpha$-tubulin surface (Gigant et al., 2014). The turn-like structure is centered on the 260-IGSTENL-266 sequence (Figure 5). This peptide 


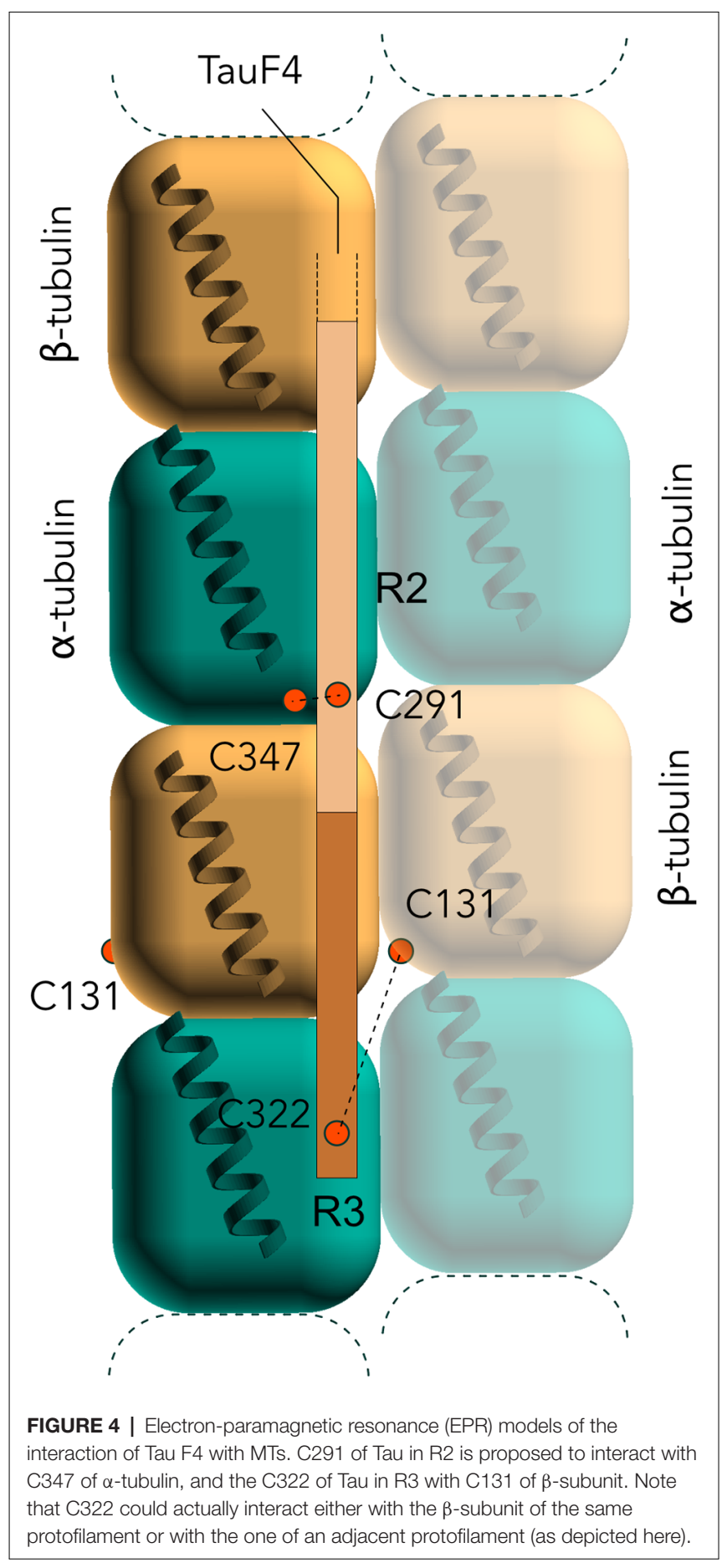

becomes immobilized in the T2R complex, as can now be explained by its position in the EM structure at the interdimer interface (Kellogg et al., 2018). The turn-like structure with a single tubulin present is not detected by smFRET (Melo et al., 2016), which is proposed to result from the different conditions of the experiments (large excess of tubulin for the smFRET or excess of Tau for the NMR experiments). The TauF4 fragment was further shown to bind, at least, at the interdimer interface as demonstrated by the competition between

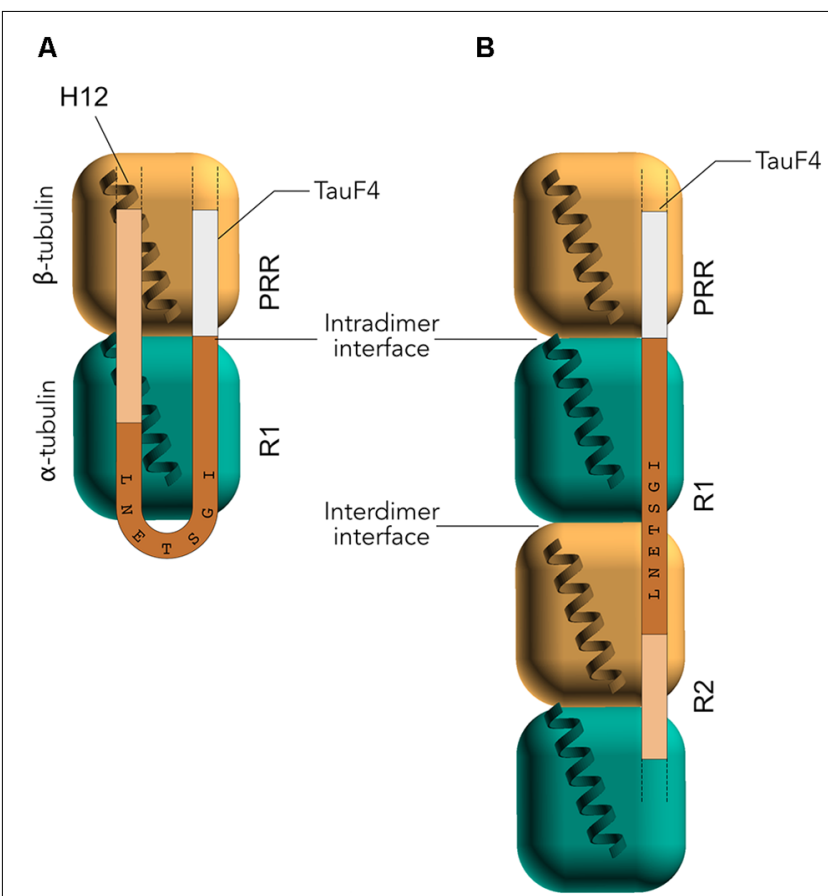

FIGURE 5 | Nuclear magnetic resonance (NMR) model of the interaction of TauF4 with stathmin-like domain (SLD)-stabilized tubulins. (A) In interaction with a single tubulin, the IGSTENL peptide of TauF4 is proposed to adopt a turn conformation and is not bound to tubulin. The PRR is mainly detected in proximity to the $\beta$-subunit. (B) In interaction with two tubulins, the IGSTENL peptide of TauF4 would be straight and overlapping two consecutive $\alpha$ - and $\beta$-subunits.

vinblastine binding and TauF4 for tubulin interaction (Kadavath et al., 2015a). The results of this study are in agreement with the stoichiometry of one Tau for the two tubulins suggested by others (Gigant et al., 2014).

Finally, the interaction between soluble tubulin and the flanking region downstream of the four Tau repeats was investigated by NMR. This region has sequence homology with the repeats and is often referred to as R' (Figure 2). By using saturation transfer difference (STD) NMR spectroscopy, two residue stretches centered on F378 and Y394 were identified in interaction with MTs (Kadavath et al., 2018).

\section{IMPACT OF TAU PHOSPHORYLATION ON ITS INTERACTION WITH MTS}

Tau-induced neurodegeneration is correlated with the appearance of Tau hyperphosphorylation, Tau aggregation into Paired Helical Filaments and/or the loss of Tau/MT binding (Hernández and Avila, 2007). Overall, phosphorylation is reported to decrease the affinity of Tau for the MTs, ensuring the dynamics of the system in healthy neurons (Lindwall and Cole, 1984; Mandelkow et al., 1995). Specific phosphorylation such as the phosphorylation of S214 (by PKA kinase) or of S262/356 (by MARK kinase) have been described to strongly decrease the affinity of Tau for MTs (Biernat et al., 1993; Scott et al., 1993; Sengupta et al., 1998; 
Schneider et al., 1999). S262 makes hydrogen bonds with a-tubulin E434, thus explaining why phosphorylation of S262 residue strongly decreases MT binding (Kellogg et al., 2018). Since phosphorylation of S262 of the isolated R1 repeat peptide does not affect its affinity for MTs, it is likely that the consequences of S262 phosphorylation on Tau binding to tubulin are due to intramolecular rearrangements of the Tau protein (Devred et al., 2002). Interestingly, phosphorylation of S214 of Tau does not significantly affect the MT assembly capacity, despite the decreased affinity (Sillen et al., 2007). Phosphorylation of S214 could also have an indirect role in vivo, as this specific phosphorylation might play a role in the observed competition between MTs and the 14-3-3 proteins for Tau binding (Hashiguchi et al., 2000). Indeed, 14-3-3 protein binding to Tau is favored when S214 is phosphorylated (Tugaeva et al., 2017), which seems to result in neurite degeneration in neuronal cell cultures (Joo et al., 2015). The 14-3-3 protein family interacts mainly with phosphorylated protein partners and is especially abundant in brain tissue (Boston et al., 1982). The 14-3-3 proteins have been implicated in a variety of human diseases, including neurodegenerative diseases. Indeed, 14-3-3 proteins are abundant in the intraneuronal deposits of aggregated Tau (Layfield et al., 1996; Umahara et al., 2004; Qureshi et al., 2013).

When Tau is phosphorylated by the CDK2/CycA3 kinase in vitro, phosphorylation at S202/T205 and T231/S235 sites are identified by NMR. Even though these phosphorylations do not significantly affect Tau binding to MTs (Amniai et al., 2009), Tau loses its capacity to assemble tubulin into MTs when at least three out of four positions are phosphorylated. This data shows that a decreased capacity of Tau to assemble tubulin into MTs, such as observed for the CDK-phosphorylated Tau, cannot be explained solely by a decreased affinity for MT surface. Additional experiments, using the shortest Tau isoform (Tau 0N3R) with T231E and S235E mutations as pseudophosphorylations, confirmed that E231/E235 do not by themselves abolish the interaction of Tau with the MTs (Schwalbe et al., 2015). However, NMR signals corresponding to residues in the PRR were less attenuated upon addition of MTs to the mutated Tau 0N3R rather than to the wild-type Tau (Schwalbe et al., 2015). This indicates that the pseudophosphorylated Tau 0N3R was locally less tightly bound to the MTs. In addition, based on the models of the Tau(225-246) peptide phosphorylated on T231 and S235, issued from comprehensive calculations of NMR parameters (distances measurements and $\mathrm{H}-\mathrm{N}$ orientations), the distances between the phosphate and the nitrogen in the directly preceding basic groups (R230 or K234, respectively) are less than $4.5 \AA$, a distance compatible with the formation of a salt-bridge. The salt bridge proposed to link phosphorylated T231 and R230 side-chains of Tau could compete with a salt bridge formation with the MTs, participating in the effect of the phosphorylation of T231 (Schwalbe et al., 2015).

Finally, Pin1 peptidyl-prolyl cis/trans isomerase was proposed to restore the capacity of CDK-phosphorylated Tau to bind to the MTs and restore MT assembly (Lu et al., 1999). However, this model was recently challenged as Pin 1 does not promote in vitro formation of phosphorylated Tau-induced MTs (Kutter et al., 2016).
For repeat R', the impact of phosphorylation on the binding affinity was assessed by means of STD NMR. Both phosphorylated Y394 and S396 were proved to weaken the interaction between Tau and MTs. However, by measuring residue-specific $\mathrm{Kd}$ values by $\mathrm{STD}$, phosphorylation on S396 had a more pronounced effect than phosphorylation on Y394, despite the fact that they are only one residue apart (Kadavath et al., 2018).

Thus, Tau phosphorylation can have an effect on MT binding and assembly through several molecular mechanisms, including electrostatic perturbations, alteration/destabilization of Tau regions bound to the MTs, a disruption of hydrogenbonds or salt-bridges and changes in structural parameters.

\section{IMPACT OF TAU ACETYLATION ON ITS INTERACTION WITH MTS}

Another Tau PTM reported to contribute to Tau pathology is lysine acetylation (Min et al., 2010, 2015); indeed, acetylated Tau is proposed as a marker of AD (Irwin et al., 2012, 2013). In this case, Lys residues are modified by the addition of an acetyl group on the $\mathrm{NH}_{3}$ moiety of their side chains, neutralizing their positive charges and modifying their steric characteristics. Consequently, acetylation affects Tau binding to MTs and impairs MT assembly (Cohen et al., 2011). Mass spectrometry (MS) analysis revealed that 14 Lys residues, mainly located in the MTBR, were acetylated in Tau samples purified from healthy mice (Morris et al., 2015). Analysis of acetylation sites obtained by in vitro acetylation with recombinant p300 acetyltransferase, by both MS and NMR (Kamah et al., 2014), identified as many as 23 modified-Lys residues. An acetylation mimicking mutation K274Q inhibits Tau tubulin polymerization ability and stimulates Tau aggregation in vitro (Rane et al., 2019). Interestingly, acetylation of K280 was detected in brain tissues from patients affected with $\mathrm{AD}$ and not in healthy brain tissues (Cohen et al., 2011). Similarly, Tau acetylation at K174 is reported as an early change in $\mathrm{AD}$ brain and in transgenic mice expressing Tau with the P301S mutation (PS19 transgenic mice; Min et al., 2015).

In addition, cross-talks between acetylation and phosphorylation modifications of Tau interplay in their regulatory role of MT dynamics (Carlomagno et al., 2017). Acetylation of K321 prevents the phosphorylation of S324, the latter being reported to inhibit Tau function of tubulin polymerization in in vitro assays (Carlomagno et al., 2017). Interestingly, this phosphorylation is one of the few modifications of Tau (among 63 modifications) specifically present in a human amyloid precursor protein transgenic $\mathrm{AD}$ mouse model when compared to a wild type mouse (Morris et al., 2015). Furthermore, phosphorylation of S324 is detected in post-mortem tissues of AD patients, but not in control samples (Carlomagno et al., 2017). Pseudo-phosphorylated mutants S324D and S324E have a diminished capacity to polymerize MTs from tubulin in in vitro assays. Similarly, acetylation of K259/K353 prevents phosphorylation of S262/S356 by the MARK kinase. Modulation of Tau acetylation could be a new strategy to inhibit Tau-mediated neurodegeneration. 
Indeed, studies in mice suggest that this is a valid diseasemodifying target. Increasing acetylation of Tau by deleting SIRT1 deacetylase in a TauP301S transgenic mouse model aggravates synapse loss, while SIRT1 overexpression limits Tau pathology propagation (Min et al., 2018). In the PS19 FTD transgenic mouse model, inhibition of p300 acetyltransferase activity lowers total Tau and K174-acetylated Tau levels. P300 inhibition prevents hippocampal atrophy and rescues memory deficits (Min et al., 2015). However, the complexity of the regulation of Tau function by PTMs has to be kept in mind. Acetylation can have both a direct inhibitory effect on Tau function and an indirect activation effect, by preventing phosphorylation in the KXGS motifs of the MTBR.

\section{IMPACT OF TAU FTD MUTATIONS ON ITS INTERACTION WITH MTS}

Tau protein is encoded by the MAPT gene located on chromosome 17. Pathogenic variants in this gene cause several related neurodegenerative diseases characterized by the presence of hyperphosphorylated Tau aggregates in the neurons (Wilhelmsen et al., 1994; D’Souza et al., 1999). Animal models with these mutations, such as P301L, have been extensively studied and are considered AD models (Lee et al., 2005). The FTD mutations of the MAPT gene alter Tau biochemical properties and/or the ratio of Tau isoforms (4R/3R ratio). Change in the isoform ratio has an indirect impact on MT assembly and the dynamics of the MT networks because the 3R Tau is known to have a lower capacity of MT stabilization and tubulin polymerization than the 4R Tau (Scott et al., 1991; Panda et al., 2003). The influence on Tau PTMs of the FTD mutations represents another indirect effect on Tau/MT interaction. The R406W Tau mutation is, for example, consistently reported to diminish Tau phosphorylation (Dayanandan et al., 1999; Matsumura et al., 1999; Delobel et al., 2002). However, almost all the FTD mutations also directly affect the ability of Tau to bind MTs and to promote tubulin assembly (Hasegawa et al., 1998; Hong et al., 1998). Tau with P301L, V337M or R406W mutations has a reduced binding to MTs and a decrease efficiency to initiate tubulin assembly. For P301L Tau, the initiation rate is decreased but the tubulin polymerization reaches the same extent in in vitro assays. Surprisingly, R406W Tau is reported to be the most affected, despite the fact that the mutation is not near the MTBR (Hong et al., 1998). This suggests that an alternative conformation might be involved. Later studies confirm the initial finding that FTD mutations affect the ability of Tau to bind MTs and to promote tubulin assembly in in vitro assays. However, there is no agreement on the extent of the specific effect of each of these mutations (Barghorn et al., 2000; DeTure et al., 2000; Combs and Gamblin, 2012). The effect of R5L, P301L, and R406W mutations of Tau differ in regard to their impact on Tau MT stabilizing capacity, not only based on their localization in Tau sequence, but also depending on the number of $\mathrm{N}$-terminal inserts $(0,1$ or $2 \mathrm{~N}$ isoforms), in the three considered 4R-Tau isoforms (Mutreja et al., 2019). In particular, Tau with the R5L mutation has a reduced ability to polymerize tubulin, with lower tubulin polymerization extent, lower rate, and longer lag time specifically for the $0 \mathrm{~N} 4 \mathrm{R}$ Tau isoform, compared to the $1 \mathrm{~N}$ and $2 \mathrm{~N}$ that are behaving as the wild-type Tau (Mutreja et al., 2019). For the P301L mutation, the Tau-dependent defect in MTs seems to diminish with the removal of each $\mathrm{N}$-terminal insert. This might be due to a differential effect of conformational changes induced by the mutations on the global hairpin-like conformation of Tau, which brings the N-terminal region close to the MTBR (Jeganathan et al., 2006). The impact of the FTD mutations on the MT stabilization capacity of Tau has been confirmed in intact cell context, with variable effect depending on the mutation (Delobel et al., 2002). Mammalian cells expressing Tau P301L show proportionally less Tau bound to MTs (Nagiec et al., 2001; Di Primio et al., 2017). However, a strong consensus on the impact of the FTD mutations in a cellular context has not yet been reached, since in neuroblastoma and $\mathrm{CHO}$ cells transfected with GFP-tagged Tau DNA constructs, the co-localization with MTs and generation of MT bundles were shown to be identical for both mutants and wild type Tau (DeTure et al., 2000). The site-dependent and isoform-dependent effect of the Tau mutations on MT stabilization were reproduced in COS cells transfected with 3R or 4R Tau isoforms (Sahara et al., 2000). For example, the V337M mutation has a significant effect when introduced in 3R Tau, but not in 4R Tau, showing disruption of the MT networks and diminished co-localization of Tau and tubulin. This isoform-specific effect of some of the mutations might explain part of the discrepancies reported on the impact of the FTD mutations of Tau on its MAP functions.

\section{PERSPECTIVES}

One of the proposed strategies in seeking $\mathrm{AD}$ treatment consists of compensating the loss of the MT-stabilizing Tau function (Cash et al., 2003; Brunden et al., 2009; Ballatore et al., 2011; Das and Ghosh, 2019). One path to this goal is to harness the therapeutic potential of MT-stabilizing agents, classically used in cancer therapies. This strategy to treat tauopathies was validated in vivo using Paclitaxel treatment in $\mathrm{AD}$ mouse models (Cash et al., 2003; Zhang B. et al., 2005) and in cell models (Brunden et al., 2011). Recently, Monacelli et al. (2017) provided an updated survey on the potential of repurposing cancer agents for $\mathrm{AD}$. Besides its implication in neurodegenerative disease, Tau is also involved in regulatory mechanisms linked to cancer development. For example, Tau was shown to regulate the MT-dependent migration of cancer cells (Breuzard et al., 2019). Finally, similar to stathmin, Tau level of expression modulates clinical MT-targeting agent efficiencies, such as taxanes or vinca alkaloids (Rouzier et al., 2005; Li et al., 2013; Smoter et al., 2013; Malesinski et al., 2015; Lin et al., 2018).

MT stabilizing peptides are another option chosen to restore MT stability (Quraishe et al., 2016; Mondal et al., 2018). A peptide such as the PS3 octapeptide was designed from the taxol-binding pocket of $\beta$-tubulin (Mondal et al., 2018). The advantage of this peptide strategy is the moderate peptide affinity for MTs that preserves the MT dynamic capacity, which is crucial for synaptic plasticity and memory. Consequently, PS3 stimulates MT polymerization and increases expression of acetylated tubulin 
in PC12 neuron cell cultures but has much fewer toxic effects than taxol. Other neuroprotective peptides have MT stabilizing functions thanks to their ability for interactions with MT end-binding proteins, which protect MT from depolymerization (Oz et al., 2014). The NAP/SAL peptides, which interact with EB1 through a SIP motif, prevent and reverse MT breakdown and axonal transport deficits in a Drosophila model of tauopathy (Quraishe et al., 2013, 2016).

Finally, MTs could be stabilized not by mimicking MAP function, but by modulating MT PTMs, which have a crucial role in MT dynamics. Levels of total $\alpha$-tubulin are reduced by approximately $65 \%$ in $\mathrm{AD}$-patient brains compared to age-matched control brains but the relative ratio of acetylated tubulin is increased by approximately $31 \%$ compared to the controls (Zhang F. et al., 2015). This suggests a compensatory mechanism to counteract MT reduction due to a loss of Tau stabilization because this modification characterizes stable MTs with slow dynamics (Kull and Sloboda, 2014; Szyk et al., 2014). Inhibition of histone deacetylase 6 (HDAC6), the major tubulin deacetylase, is thus an alternative strategy to compensate for Tau MAP function. Indeed, in transgenic mouse models of AD, inhibition of HDAC6 improves memory (Kilgore et al., 2010; Govindarajan et al., 2013; Selenica et al., 2014). Similarly, in Drosophila, HDAC6 null mutation rescues MT defects through increased tubulin acetylation (Xiong et al., 2013).

\section{REFERENCES}

Alonso, A. C., Zaidi, T., Grundke-Iqbal, T., and Iqbal, K. (1994). Role of abnormally phosphorylated tau in the breakdown of microtubules in Alzheimer disease. Proc Natl. Acad. Sci. U S A 91, 5562-5566. doi: 10.1073/pnas.91.12.5562

Alushin, G. M., Lander, G. C., Kellogg, E. H., Zhang, R., Baker, D., and Nogales, E. (2014). High-resolution microtubule structures reveal the structural transitions in $\alpha \beta$-tubulin upon GTP hydrolysis. Cell 157, 1117-1129. doi: 10.1016/j.cell. 2014.03.053

Amniai, L., Barbier, P., Sillen, A., Wieruszeski, J.-M., Peyrot, V., Lippens, G., et al. (2009). Alzheimer disease specific phosphoepitopes of Tau interfere with assembly of tubulin but not binding to microtubules. FASEB J. 23, 1146-1152. doi: 10.1096/fj.08-121590

Amos, L. A., and Schlieper, D. (2005). Microtubules and maps. Adv. Protein Chem. 71, 257-298. doi: 10.1016/S0065-3233(04)71007-4

Baas, P. W., and Qiang, L. (2019). Tau: it's not what you think. Trends Cell Biol. 29, 452-461. doi: 10.1016/j.tcb.2019.02.007

Baas, P. W., Rao, A. N., Matamoros, A. J., and Leo, L. (2016). Stability properties of neuronal microtubules. Cytoskelet 73, 442-460. doi: 10.1002/ cm.21286

Ballatore, C., Brunden, K. R., Trojanowski, J. Q., Lee, V. M., Smith, A. B. III., and Huryn, D. M. (2011). Modulation of protein-protein interactions as a therapeutic strategy for the treatment of neurodegenerative tauopathies. Curr. Top. Med. Chem. 11, 317-330. doi: 10.2174/15680261179 4072605

Barbier, P., Dorléans, A., Devred, F., Sanz, L., Allegro, D., Alfonso, C., et al. (2010). Stathmin and interfacial microtubule inhibitors recognize a naturally curved conformation of tubulin dimers. J. Biol. Chem. 285, 31672-31681. doi: 10.1074/jbc.m110.141929

Barghorn, S., Zheng-Fischhöfer, Q., Ackmann, M., Biernat, J., von Bergen, M., Mandelkow, E. M., et al. (2000). Structure, microtubule interactions, and paired helical filament aggregation by tau mutants of frontotemporal dementias. Biochemistry 39, 11714-11721. doi: 10.1021/bi000850r

Biernat, J., Gustke, N., Drewes, G., Mandelkow, E. M., and Mandelkow, E. (1993). Phosphorylation of Ser262 strongly reduces binding of tau to microtubules:
Overall, Tau implication in neurodegenerative diseases, and other diseases where MTs play an important role, clearly shows the interest of the Tau/MTs interaction as a potential target for intervention (Pachima et al., 2016; Das and Ghosh, 2019). Many advances have been made in the understanding of Tau functions as a MAP, and in the structural aspects of the Tau/MTs interaction. On this basis, the importance of regulatory factors of the interaction, from PTMs to other endogenous cofactors such as Zinc (Fichou et al., 2019), can now be addressed. This will hopefully lead to new strategies targeting disease where MTs, and consequently Tau, are involved.

\section{AUTHOR CONTRIBUTIONS}

The content of the manuscript was drafted and edited by all authors.

\section{FUNDING}

IL, CS-N, AL and OZ acknowledge support by the Laboratory of Excellence (LabEx, Agence Nationale de la Recherche-ANR), Development of Innovative Strategies for a Transdisciplinary approach to Alzheimer's disease (DISTALZ), Lille Excellence Centre for Neurodegenerative Disease (LiCEND) and the project TUNABLE from the I-site ULNE (Lille University).

distinction between PHF-like immunoreactivity and microtubule binding. Neuron 11, 153-163. doi: 10.1016/0896-6273(93)90279-Z

Binder, L. I., Frankfurter, A., and Rebhun, L. I. (1985). The distribution of tau in the mammalian central nervous system. J. Cell Biol. 101, 1371-1378. doi: $10.1083 /$ jcb.101.4.1371

Black, M. M., Slaughter, T., Moshiach, S., Obrocka, M., and Fischer, I. (1996). Tau is enriched on dynamic microtubules in the distal region of growing axons. J. Neurosci. 16, 3601-3619. doi: 10.1523/JNEUROSCI.16-11-03601.1996

Boston, P. F., Jackson, P., and Thompson, R. J. (1982). Human 14-3-3 protein: radioimmunoassay, tissue distribution, and cerebrospinal fluid levels in patients with neurological disorders. J. Neurochem. 38, 1475-1482. doi: 10.1111/j.1471-4159.1982.tb07928.x

Bowne-Anderson, H., Hibbel, A., and Howard, J. (2015). Regulation of microtubule growth and catastrophe: unifying theory and experiment. Trends Cell Biol. 25, 769-779. doi: 10.1016/j.tcb.2015.08.009

Breuzard, G., Hubert, P., Nouar, R., De Bessa, T., Devred, F., Barbier, P., et al. (2013). Molecular mechanisms of Tau binding to microtubules and its role in microtubule dynamics in live cells. J. Cell Sci. 126, 2810-2819. doi: 10.1242/jcs. 120832

Breuzard, G., Pagano, A., Bastonero, S., Malesinski, S., Parat, F., Barbier, P., et al. (2019). Tau regulates the microtubule-dependent migration of glioblastoma cells via the Rho-ROCK signaling pathway. J. Cell Sci. 132:jcs222851. doi: $10.1242 /$ jcs.222851

Brion, J. P., Flament-Durand, J., and Dustin, P. (1986). Alzheimer's disease and tau proteins. Lancet 2:1098. doi: 10.1016/s0140-6736(86)90495-2

Brunden, K. R., Trojanowski, J. Q., and Lee, V. M. (2009). Advances in tau-focused drug discovery for Alzheimer's disease and related tauopathies. Nat. Rev. Drug Discov. 8, 783-793. doi: 10.1038/nrd2959

Brunden, K. R., Yao, Y., Potuzak, J. S., Ferrer, N. I., Ballatore, C., James, M. J., et al. (2011). The characterization of microtubule-stabilizing drugs as possible therapeutic agents for Alzheimer's disease and related tauopathies. Pharmacol. Res. 63, 341-351. doi: 10.1016/j.phrs.2010.12.002

Butner, K. A., and Kirschner, M. W. (1991). Tau protein binds to microtubules through a flexible array of distributed weak sites. J. Cell Biol. 115, 717-730. doi: $10.1083 /$ jcb.115.3.717 
Caplow, M., and Shanks, J. (1990). Mechanism of the microtubule GTPase reaction. J. Biol. Chem. 265, 8935-8941.

Carlier, M. F., Didry, D., Simon, C., and Pantaloni, D. (1989). Mechanism of GTP hydrolysis in tubulin polymerization: characterization of the kinetic intermediate microtubule-GDP-Pi using phosphate analogues. Biochemistry 28, 1783-1791. doi: 10.1021/bi00430a054

Carlomagno, Y., Chung, D.-E. C., Yue, M., Castanedes-Casey, M., Madden, B. J., Dunmore, J., et al. (2017). An acetylation-phosphorylation switch that regulates tau aggregation propensity and function. J. Biol. Chem. 292, 15277-15286. doi: 10.1074/jbc.M117.794602

Cash, A. D., Aliev, G., Siedlak, S. L., Nunomura, A., Fujioka, H., Zhu, X., et al. (2003). Microtubule reduction in Alzheimer's disease and aging is independent of tau filament formation. Am. J. Pathol. 162, 1623-1627. doi: 10.1016/s00029440(10)64296-4

Chen, J., Kanai, Y., Cowan, N. J., and Hirokawa, N. (1992). Projection domains of MAP2 and tau determine spacings between microtubules in dendrites and axons. Nature 360, 674-677. doi: 10.1038/360674a0

Chrétien, D., Fuller, S. D., and Karsenti, E. (1995). Structure of growing microtubule ends: two-dimensional sheets close into tubes at variable rates. J. Cell Biol. 129, 1311-1328. doi: 10.1083/jcb.129.5.1311

Cohen, T. J., Guo, J. L., Hurtado, D. E., Kwong, L. K., Mills, I. P., Trojanowski, J. Q., et al. (2011). The acetylation of tau inhibits its function and promotes pathological tau aggregation. Nat. Commun. 2:252. doi: 10.1038/ ncomms 1255

Combs, B., and Gamblin, T. C. (2012). FTDP-17 tau mutations induce distinct effects on aggregation and microtubule interactions. Biochemistry 51, 8597-8607. doi: 10.1021/bi3010818

Cummings, J., Lee, G., Mortsdorf, T., Ritter, A., and Zhong, K. (2017). Alzheimer's disease drug development pipeline: 2017. Alzheimers Dement. 3, 367-384. doi: 10.1016/j.trci.2017.05.002

D’Souza, I., Poorkaj, P., Hong, M., Nochlin, D., Lee, V. M., Bird, T. D., et al. (1999). Missense and silent tau gene mutations cause frontotemporal dementia with parkinsonism-chromosome 17 type, by affecting multiple alternative RNA splicing regulatory elements. Proc. Natl. Acad. Sci. U S A 96, 5598-5603. doi: $10.1073 /$ pnas.96.10.5598

Das, G., and Ghosh, S. (2019). Why microtubules should be considered as one of the supplementary targets for designing neurotherapeutics. ACS Chem. Neurosci. 10, 1118-1120. doi: 10.1021/acschemneuro. $9 \mathrm{~b} 00002$

Dayanandan, R., Van Slegtenhorst, M., Mack, T. G., Ko, L., Yen, S. H., Leroy, K., et al. (1999). Mutations in tau reduce its microtubule binding properties in intact cells and affect its phosphorylation. FEBS Lett. 446, 228-232. doi: 10.1016/s0014-5793(99)00222-7

Delobel, P., Flament, S., Hamdane, M., Jakes, R., Rousseau, A., Delacourte, A., et al. (2002). Functional characterization of FTDP-17 tau gene mutations through their effects on Xenopus oocyte maturation. J. Biol. Chem. 277, 9199-9205. doi: 10.1074/jbc.M107716200

DeTure, M., Ko, L. W., Yen, S., Nacharaju, P., Easson, C., Lewis, J., et al. (2000). Missense tau mutations identified in FTDP-17 have a small effect on tau-microtubule interactions. Brain Res. 853, 5-14. doi: 10.1016/s00068993(99)02124-1

Devred, F., Barbier, P., Douillard, S., Monasterio, O., Andreu, J. M., and Peyrot, V. (2004). Tau induces ring and microtubule formation from $\alpha \beta$ tubulin dimers under nonassembly conditions. Biochemistry 43, 10520-10531. doi: 10.1021/bi0493160

Devred, F., Barbier, P., Lafitte, D., Landrieu, I., Lippens, G., and Peyrot, V. (2010). Microtubule and MAPs: thermodynamics of complex formation by AUC, ITC, fluorescence, and NMR. Methods Cell Biol. 95, 449-480. doi: 10.1016/S0091679X(10)95023-1

Devred, F., Douillard, S., Briand, C., and Peyrot, V. (2002). First tau repeat domain binding to growing and taxol-stabilized microtubules and serine 262 residue phosphorylation. FEBS Lett. 523, 247-251. doi: 10.1016/s0014-5793(02) 02999-x

Di Maio, I. L., Barbier, P., Allegro, D., Brault, C., and Peyrot, V. (2014). Quantitative analysis of tau-microtubule interaction using FRET. Int. J. Mol. Sci. 15, 14697-14714. doi: 10.3390/ijms150814697

Di Primio, C., Quercioli, V., Siano, G., Rovere, M., Kovacech, B., Novak, M., et al. (2017). The distance between $\mathrm{N}$ and $\mathrm{C}$ termini of Tau and of FTDP-17 mutants is modulated by microtubule interactions in living cells. Front. Mol. Neurosci. 10:210. doi: 10.3389/fnmol.2017.00210

Dompierre, J. P., Godin, J. D., Charrin, B. C., Cordelières, F. P., King, S. J., Humbert, S., et al. (2007). Histone deacetylase 6 inhibition compensates for the transport deficit in Huntington's disease by increasing tubulin acetylation. J. Neurosci. 27, 3571-3583. doi: 10.1523/JNEUROSCI.0037 $-07.2007$

Donhauser, Z. J., Saunders, J. T., D'Urso, D. S., and Garrett, T. A. (2017). Dimerization and long-range repulsion established by both termini of the microtubule-associated protein Tau. Biochemistry 56, 5900-5909. doi: 10.1021/acs.biochem.7b00653

Duan, A. R., Jonasson, E. M., Alberico, E. O., Li, C., Scripture, J. P., Miller, R. A., et al. (2017). Interactions between Tau and different conformations of tubulin: implications for Tau function and mechanism. J. Mol. Biol. 429, 1424-1438. doi: 10.1016/j.jmb.2017.03.018

Eftekharzadeh, B., Daigle, J. G., Kapinos, L. E., Coyne, A., Schiantarelli, J., Carlomagno, Y., et al. (2018). Tau protein disrupts nucleocytoplasmic transport in Alzheimer's disease. Neuron 99, 925.e7-940.e7. doi: 10.1016/j.neuron.2018. 07.039

Fauquant, C., Redeker, V., Landrieu, I., Wieruszeski, J. M., Verdegem, D., Laprevote, O., et al. (2011). Systematic identification of tubulin-interacting fragments of the microtubule-associated protein Tau leads to a highly efficient promoter of microtubule assembly. J. Biol. Chem. 286, 33358-33368. doi: 10.1074/jbc.m111.223545

Fichou, Y., Al-Hilaly, Y. K., Devred, F., Smet-Nocca, C., Tsvetkov, P. O., Verelst, J., et al. (2019). The elusive tau molecular structures: can we translate the recent breakthroughs into new targets for intervention? Acta Neuropathol. Commun. 7:31. doi: 10.1186/s40478-019-0682-X

Gadadhar, S., Bodakuntla, S., Natarajan, K., and Janke, C. (2017). The tubulin code at a glance. J. Cell Sci. 130, 1347-1353. doi: 10.1242/jcs.199471

Gigant, B., Curmi, P. A., Martin-Barbey, C., Charbaut, E., Lachkar, S., Lebeau, L., et al. (2000). The 4 A X-ray structure of a tubulin:stathminlike domain complex. Cell 102, 809-816. doi: 10.1016/s0092-8674(00) 00069-6

Gigant, B., Landrieu, I., Fauquant, C., Barbier, P., Huvent, I., Wieruszeski, J.-M., et al. (2014). Mechanism of Tau-promoted microtubule assembly as probed by NMR spectroscopy. J. Am. Chem. Soc. 136, 12615-12623. doi: $10.1021 /$ ja504864m

Godena, V. K., Brookes-Hocking, N., Moller, A., Shaw, G., Oswald, M., Sancho, R. M., et al. (2014). Increasing microtubule acetylation rescues axonal transport and locomotor deficits caused by LRRK2 Roc-COR domain mutations. Nat. Commun. 5:5245. doi: 10.1038/ncomms6245

Goedert, M., Spillantini, M. G., Jakes, R., Rutherford, D., and Crowther, R. A. (1989). Multiple isoforms of human microtubule-associated protein tau: sequences and localization in neurofibrillary tangles of Alzheimer's disease. Neuron 3, 519-526. doi: 10.1016/0896-6273(89)90210-9

Goode, B. L., Chau, M., Denis, P. E., and Feinstein, S. C. (2000). Structural and functional differences between 3-repeat and 4-repeat tau isoforms. Implications for normal tau function and the onset of neurodegenetative disease. J. Biol. Chem. 275, 38182-38189. doi: 10.1074/jbc.m007489200

Goode, B. L., Denis, P. E., Panda, D., Radeke, M. J., Miller, H. P., Wilson, L., et al. (1997). Functional interactions between the proline-rich and repeat regions of tau enhance microtubule binding and assembly. Mol. Biol. Cell 8, 353-365. doi: $10.1091 / \mathrm{mbc} .8 .2 .353$

Govindarajan, N., Rao, P., Burkhardt, S., Sananbenesi, F., Schlüter, O. M., Bradke, F., et al. (2013). Reducing HDAC6 ameliorates cognitive deficits in a mouse model for Alzheimer's disease. EMBO Mol. Med. 5, 52-63. doi: 10.1002/emmm.201201923

Guo, W., Naujock, M., Fumagalli, L., Vandoorne, T., Baatsen, P., Boon, R., et al. (2017). HDAC6 inhibition reverses axonal transport defects in motor neurons derived from FUS-ALS patients. Nat. Commun. 8:861. doi: 10.1038/s41467017-00911-y

Gustke, N., Trinczek, B., Biernat, J., Mandelkow, E. M., and Mandelkow, E. (1994). Domains of tau protein and interactions with microtubules. Biochemistry 33 , 9511-9522. doi: 10.1021/bi00198a017

Hasegawa, M., Smith, M. J., and Goedert, M. (1998). Tau proteins with FTDP-17 mutations have a reduced ability to promote microtubule assembly. FEBS Lett. 437, 207-210. doi: 10.1016/s0014-5793(98)01217-4 


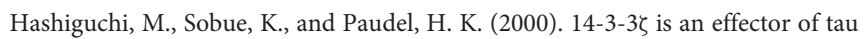
protein phosphorylation. J. Biol. Chem. 275, 25247-25254. doi: 10.1074/jbc. m003738200

Hernández, F., and Avila, J. (2007). Tauopathies. Cell. Mol. Life Sci. 64, 2219-2233. doi: 10.1007/s00018-007-7220-x

Hernández-Vega, A., Braun, M., Scharrel, L., Jahnel, M., Wegmann, S., Hyman, B. T., et al. (2017). Local nucleation of microtubule bundles through tubulin concentration into a condensed Tau phase. Cell Rep. 20, 2304-2312. doi: 10.1016/j.celrep.2017.08.042

Himmler, A., Drechsel, D., Kirschner, M. W., and Martin, D. W. Jr. (1989). Tau consists of a set of proteins with repeated C-terminal microtubule-binding domains and variable N-terminal domains. Mol. Cell. Biol. 9, 1381-1388. doi: $10.1128 / \mathrm{mcb} .9 .4 .1381$

Hinrichs, M. H., Jalal, A., Brenner, B., Mandelkow, E., Kumar, S., and Scholz, T. (2012). Tau protein diffuses along the microtubule lattice. J. Biol. Chem. 287, 38559-38568. doi: 10.1074/jbc.m112.369785

Hong, M., Zhukareva, V., Vogelsberg-Ragaglia, V., Wszolek, Z., Reed, L., Miller, B. I., et al. (1998). Mutation-specific functional impairments in distinct tau isoforms of hereditary FTDP-17. Science 282, 1914-1917. doi: 10.1126/science.282.5395.1914

Howard, W. D., and Timasheff, S. N. (1986). GDP state of tubulin: stabilization of double rings. Biochemistry 25, 8292-8300. doi: 10.1021/bi00373a025

Irwin, D. J., Cohen, T. J., Grossman, M., Arnold, S. E., McCarty-Wood, E., Van Deerlin, V. M., et al. (2013). Acetylated tau neuropathology in sporadic and hereditary tauopathies. Am. J. Pathol. 183, 344-351. doi: 10.1016/j.ajpath.2013. 04.025

Irwin, D. J., Cohen, T. J., Grossman, M., Arnold, S. E., Xie, S. X., Lee, V. M., et al. (2012). Acetylated tau, a novel pathological signature in Alzheimer's disease and other tauopathies. Brain 135, 807-818. doi: 10.1093/brain/ aws013

Jadhav, S., Avila, J., Schöll, M., Kovacs, G. G., Kövari, E., Skrabana, R., et al. (2019). A walk through tau therapeutic strategies. Acta Neuropathol. Commun. 7:22. doi: 10.1186/s40478-019-0664-z

Janning, D., Igaev, M., Sündermann, F., Brühmann, J., Beutel, O., Heinisch, J. J., et al. (2014). Single-molecule tracking of tau reveals fast kiss-and-hop interaction with microtubules in living neurons. Mol. Biol. Cell 25, 3541-3551. doi: 10.1091/mbc.e14-06-1099

Jeganathan, S., von Bergen, M., Brutlach, H., Steinhoff, H. J., and Mandelkow, E. (2006). Global hairpin folding of tau in solution. Biochemistry 45, 2283-2293. doi: 10.1021/bi0521543

Joo, Y., Schumacher, B., Landrieu, I., Bartel, M., Smet-Nocca, C., Jang, A., et al. (2015). Involvement of 14-3-3 in tubulin instability and impaired axon development is mediated by Tau. FASEB J. 29, 4133-4144. doi: 10.1096/fj.14265009

Kadavath, H., Cabrales Fontela, Y., Jaremko, M., Jaremko, €., Overkamp, K., Biernat, J., et al. (2018). The binding mode of a Tau peptide with tubulin. Angew. Chem. Int. Ed Engl. 57, 3246-3250. doi: 10.1002/anie.201712089

Kadavath, H., Hofele, R. V., Biernat, J., Kumar, S., Tepper, K., Urlaub, H., et al. (2015a). Tau stabilizes microtubules by binding at the interface between tubulin heterodimers. Proc. Natl. Acad. Sci. U S A 112, 7501-7506. doi: 10.1073/pnas. 1504081112

Kadavath, H., Jaremko, M., Jaremko, €., Biernat, J., Mandelkow, E., and Zweckstetter, M. (2015b). Folding of the Tau protein on microtubules. Angew. Chem. Int. Ed Engl. 54, 10347-10351. doi: 10.1002/anie.201501714

Kamah, A., Huvent, I., Cantrelle, F.-X., Qi, H., Lippens, G., Landrieu, I., et al. (2014). Nuclear magnetic resonance analysis of the acetylation pattern of the neuronal Tau protein. Biochemistry 53, 3020-3032. doi: 10.1021/ bi500006v

Kar, S., Fan, J., Smith, M. J., Goedert, M., and Amos, L. A. (2003). Repeat motifs of tau bind to the insides of microtubules in the absence of taxol. EMBO J. 22, 70-77. doi: 10.1093/emboj/cdg001

Kellogg, E. H., Hejab, N. M. A., Poepsel, S., Downing, K. H., DiMaio, F., and Nogales, E. (2018). Near-atomic model of microtubule-tau interactions. Science 360, 1242-1246. doi: 10.1126/science.aat1780

Kilgore, M., Miller, C. A., Fass, D. M., Hennig, K. M., Haggarty, S. J., Sweatt, J. D., et al. (2010). Inhibitors of class 1 histone deacetylases reverse contextual memory deficits in a mouse model of Alzheimer's disease. Neuropsychopharmacology 35, 870-880. doi: 10.1038/npp.2009.197
Kull, F. J., and Sloboda, R. D. (2014). A slow dance for microtubule acetylation. Cell 157, 1255-1256. doi: 10.1016/j.cell.2014.05.021

Kutter, S., Eichner, T., Deaconescu, A. M., and Kern, D. (2016). Regulation of microtubule assembly by tau and not by Pin1. J. Mol. Biol. 428, 1742-1759. doi: 10.1016/j.jmb.2016.03.010

Layfield, R., Fergusson, J., Aitken, A., Lowe, J., Landon, M., and Mayer, R. J. (1996). Neurofibrillary tangles of Alzheimer's disease brains contain 14-3-3 proteins. Neurosci. Lett. 209, 57-60. doi: 10.1016/0304-3940(96)12598-2

LeBoeuf, A. C., Levy, S. F., Gaylord, M., Bhattacharya, A., Singh, A. K., Jordan, M. A., et al. (2008). FTDP-17 mutations in Tau alter the regulation of microtubule dynamics: an "alternative core" model for normal and pathological Tau action. J. Biol. Chem. 283, 36406-36415. doi: 10.1074/jbc.M803519200

Lee, V. M.-Y., Kenyon, T. K., and Trojanowski, J. Q. (2005). Transgenic animal models of tauopathies. Biochim. Biophys. Acta 1739, 251-259. doi: 10.1016/j. bbadis.2004.06.014

Li, X.-H., Culver, J. A., and Rhoades, E. (2015). Tau binds to multiple tubulin dimers with helical structure. J. Am. Chem. Soc. 137, 9218-9221. doi: $10.1021 /$ jacs.5b04561

Li, Z.-H., Xiong, Q.-Y., Tu, J.-H., Gong, Y., Qiu, W., Zhang, H.-Q., et al. (2013). Tau proteins expressions in advanced breast cancer and its significance in taxane-containing neoadjuvant chemotherapy. Med. Oncol. 30:591. doi: 10.1007/s12032-013-0591-y

Lin, H., Zheng, L., Li, S., Xie, B., Cui, B., Xia, A., et al. (2018). Cytotoxicity of Tanshinone IIA combined with Taxol on drug-resist breast cancer cells MCF-7 through inhibition of Tau. Phytother. Res. 32, 667-671. doi: 10.1002/ptr.6014

Lindwall, G., and Cole, R. D. (1984). Phosphorylation affects the ability of tau protein to promote microtubule assembly. J. Biol. Chem. 259, 5301-5305.

Lu, P. J., Wulf, G., Zhou, X. Z., Davies, P., and Lu, K. P. (1999). The prolyl isomerase Pin 1 restores the function of Alzheimer-associated phosphorylated tau protein. Nature 399, 784-788. doi: 10.1038/21650

Magiera, M. M., Bodakuntla, S., Žiak, J., Lacomme, S., Marques Sousa, P., Leboucher, S., et al. (2018). Excessive tubulin polyglutamylation causes neurodegeneration and perturbs neuronal transport. EMBO J. 37:e100440. doi: 10.15252/embj.2018100440

Makrides, V., Massie, M. R., Feinstein, S. C., and Lew, J. (2004). Evidence for two distinct binding sites for tau on microtubules. Proc. Natl. Acad. Sci. U S A 101, 6746-6751. doi: 10.1073/pnas.0400992101

Malesinski, S., Tsvetkov, P. O., Kruczynski, A., Peyrot, V., and Devred, F. (2015). Stathmin potentiates vinflunine and inhibits Paclitaxel activity. PLoS One 10:e0128704. doi: 10.1371/journal.pone.0128704

Mandelkow, E. M., Biernat, J., Drewes, G., Gustke, N., Trinczek, B., and Mandelkow, E. (1995). Tau domains, phosphorylation and interactions with microtubules. Neurobiol. Aging 16, 355-362; discussion 362-363. doi: 10.1016/0197-4580(95)00025-a

Martinho, M., Allegro, D., Huvent, I., Chabaud, C., Etienne, E., Kovacic, H., et al. (2018). Two Tau binding sites on tubulin revealed by thiol-disulfide exchanges. Sci. Rep. 8:13846. doi: 10.1038/s41598-018-32096-9

Matsumura, N., Yamazaki, T., and Ihara, Y. (1999). Stable expression in Chinese hamster ovary cells of mutated tau genes causing frontotemporal dementia and parkinsonism linked to chromosome 17 (FTDP-17). Am. J. Pathol. 154, 1649-1656. doi: 10.1016/s0002-9440(10)65420-x

Melki, R., Carlier, M. F., Pantaloni, D., and Timasheff, S. N. (1989). Cold depolymerization of microtubules to double rings: geometric stabilization of assemblies. Biochemistry 28, 9143-9152. doi: 10.1021/bi00449a028

Melková, K., Zapletal, V., Narasimhan, S., Jansen, S., Hritz, J., Škrabana, R., et al. (2019). Structure and functions of microtubule associated proteins tau and MAP2c: similarities and differences. Biomolecules 9:E105. doi: 10.3390/biom9030105

Melo, A. M., Coraor, J., Alpha-Cobb, G., Elbaum-Garfinkle, S., Nath, A., and Rhoades, E. (2016). A functional role for intrinsic disorder in the tau-tubulin complex. Proc. Natl. Acad. Sci. U S A 113, 14336-14341. doi: 10.1073/pnas. 1610137113

Min, S.-W., Chen, X., Tracy, T. E., Li, Y., Zhou, Y., Wang, C., et al. (2015). Critical role of acetylation in tau-mediated neurodegeneration and cognitive deficits. Nat. Med. 21, 1154-1162. doi: 10.1038/nm.3951

Min, S. W., Cho, S. H., Zhou, Y., Schroeder, S., Haroutunian, V., Seeley, W. W., et al. (2010). Acetylation of tau inhibits its degradation 
and contributes to tauopathy. Neuron 67, 953-966. doi: 10.1016/j.neuron.2010. 08.044

Min, S.-W., Sohn, P. D., Li, Y., Devidze, N., Johnson, J. R., Krogan, N. J., et al. (2018). SIRT1 deacetylates tau and reduces pathogenic tau spread in a mouse model of tauopathy. J. Neurosci. 38, 3680-3688. doi: 10.1523/jneurosci.236917.2018

Mitchison, T., and Kirschner, M. (1984). Dynamic instability of microtubule growth. Nature 312, 237-242. doi: 10.1038/312237a0

Monacelli, F., Cea, M., Borghi, R., Odetti, P., and Nencioni, A. (2017). Do cancer drugs counteract neurodegeneration? Repurposing for Alzheimer's disease. J. Alzheimers Dis. 55, 1295-1306. doi: 10.3233/jad-160840

Mondal, P., Das, G., Khan, J., Pradhan, K., and Ghosh, S. (2018). Crafting of neuroprotective octapeptide from taxol-binding pocket of $\beta$-tubulin. ACS Chem. Neurosci. 9, 615-625. doi: 10.1021/acschemneuro.7b 00457

Morris, M., Knudsen, G. M., Maeda, S., Trinidad, J. C., Ioanoviciu, A., Burlingame, A. L., et al. (2015). Tau post-translational modifications in wild-type and human amyloid precursor protein transgenic mice. Nat. Neurosci. 18, 1183-1189. doi: 10.1038/nn.4067

Mudher, A., Brion, J.-P., Avila, J., Medina, M., and Buée, L. (2017). EuroTau: towing scientists to tau without tautology. Acta Neuropathol. Commun. 5:90. doi: 10.1186/s40478-017-0491-z

Mukhopadhyay, R., and Hoh, J. H. (2001). AFM force measurements on microtubule-associated proteins: the projection domain exerts a long-range repulsive force. FEBS Lett. 505, 374-378. doi: 10.1016/s0014-5793(01)02844-7

Mukrasch, M. D., Bibow, S., Korukottu, J., Jeganathan, S., Biernat, J., Griesinger, C., et al. (2009). Structural polymorphism of 441-residue tau at single residue resolution. PLoS Biol. 7:e34. doi: 10.1371/journal.pbio.1000034

Mukrasch, M. D., Markwick, P., Biernat, J., Bergen, M., Bernado, P., Griesinger, C., et al. (2007). Highly populated turn conformations in natively unfolded tau protein identified from residual dipolar couplings and molecular simulation. J. Am. Chem. Soc. 129, 5235-5243. doi: 10.1021/ja0690159

Murphy, D. B., Johnson, K. A., and Borisy, G. G. (1977). Role of tubulin-associated proteins in microtubule nucleation and elongation. J. Mol. Biol. 117, 33-52. doi: 10.1016/0022-2836(77)90021-3

Mutreja, Y., Combs, B., and Gamblin, T. C. (2019). FTDP-17 mutations alter the aggregation and microtubule stabilization propensity of tau in an isoformspecific fashion. Biochemistry 58, 742-754. doi: 10.1021/acs.biochem.8b01039

Nagiec, E. W., Sampson, K. E., and Abraham, I. (2001). Mutated tau binds less avidly to microtubules than wildtype tau in living cells. J. Neurosci. Res. 63, 268-275. doi: 10.1002/1097-4547(20010201)63:3<268::aid-jnr1020>3.0. co;2-e

Nawrotek, A., Knossow, M., and Gigant, B. (2011). The determinants that govern microtubule assembly from the atomic structure of GTP-tubulin. J. Mol. Biol. 412, 35-42. doi: 10.1016/j.jmb.2011.07.029

Nouar, R., Devred, F., Breuzard, G., and Peyrot, V. (2013). FRET and FRAP imaging: approaches to characterise tau and stathmin interactions with microtubules in cells. Biol. Cell 105, 149-161. doi: 10.1111/boc.2012 00060

Oz, S., Kapitansky, O., Ivashco-Pachima, Y., Malishkevich, A., Giladi, E., Skalka, N., et al. (2014). The NAP motif of activity-dependent neuroprotective protein $(\mathrm{ADNP})$ regulates dendritic spines through microtubule end binding proteins. Mol. Psychiatry 19, 1115-1124. doi: 10.1038/mp.2014.97

Pachima, Y. I., Zhou, L., Lei, P., and Gozes, I. (2016). Microtubule-tau interaction as a therapeutic target for Alzheimer's disease. J. Mol. Neurosci. 58, 145-152. doi: 10.1007/s12031-016-0715-x

Panda, D., Samuel, J. C., Massie, M., Feinstein, S. C., and Wilson, L. (2003). Differential regulation of microtubule dynamics by three- and four-repeat tau: implications for the onset of neurodegenerative disease. Proc. Natl. Acad. Sci. U S A 100, 9548-9553. doi: 10.1073/pnas.1633508100

Paonessa, F., Evans, L. D., Solanki, R., Larrieu, D., Wray, S., Hardy, J., et al. (2019). Microtubules deform the nuclear membrane and disrupt nucleocytoplasmic transport in tau-mediated frontotemporal dementia. Cell Rep. 26, 582.e5-593.e5. doi: 10.1016/j.celrep.2018.12.085

Pecqueur, L., Duellberg, C., Dreier, B., Jiang, Q., Wang, C., Pluckthun, A., et al. (2012). A designed ankyrin repeat protein selected to bind to tubulin caps the microtubule plus end. Proc. Natl. Acad. Sci. U S A 109, 12011-12016. doi: $10.1073 /$ pnas. 1204129109
Preuss, U., Biernat, J., Mandelkow, E. M., and Mandelkow, E. (1997). The “jaws" model of tau-microtubule interaction examined in CHO cells. J. Cell Sci. 110, $789-800$.

Qiang, L., Sun, X., Austin, T. O., Muralidharan, H., Jean, D. C., Liu, M., et al. (2018). Tau does not stabilize axonal microtubules but rather enables them to have long labile domains. Curr. Biol. 28, 2181.e4-2189.e4. doi: 10.1016/j.cub. 2018.05.045

Quraishe, S., Cowan, C. M., and Mudher, A. (2013). NAP (davunetide) rescues neuronal dysfunction in a Drosophila model of tauopathy. Mol. Psychiatry 18, 834-842. doi: $10.1038 / \mathrm{mp} .2013 .32$

Quraishe, S., Sealey, M., Cranfield, L., and Mudher, A. (2016). Microtubule stabilising peptides rescue tau phenotypes in vivo. Sci. Rep. 6:38224. doi: 10.1038/srep38224

Qureshi, H. Y., Li, T., MacDonald, R., Cho, C. M., Leclerc, N., and Paudel, H. K.

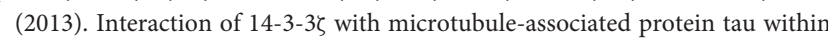
Alzheimer's disease neurofibrillary tangles. Biochemistry 52, 6445-6455. doi: 10.1021/bi400442d

Ramkumar, A., Jong, B. Y., and Ori-McKenney, K. M. (2018). ReMAPping the microtubule landscape: how phosphorylation dictates the activities of microtubule-associated proteins. Dev. Dyn. 247, 138-155. doi: 10.1002/dvdy. 24599

Rane, J. S., Kumari, A., and Panda, D. (2019). An acetylation mimicking mutation, K274Q, in tau imparts neurotoxicity by enhancing tau aggregation and inhibiting tubulin polymerization. Biochem. J. 476, 1401-1417. doi: 10.1042/bcj20190042

Roman, A. Y., Devred, F., Byrne, D., La Rocca, R., Ninkina, N. N., Peyrot, V., et al. (2019). Zinc induces temperature-dependent reversible self-assembly of tau. J. Mol. Biol. 431, 687-695. doi: 10.1016/j.jmb.2018.12.008

Rosenberg, K. J., Ross, J. L., Feinstein, H. E., Feinstein, S. C., and Israelachvili, J. (2008). Complementary dimerization of microtubule-associated tau protein: implications for microtubule bundling and tau-mediated pathogenesis. Proc. Natl. Acad. Sci. U S A 105, 7445-7450. doi: 10.1073/pnas.08020 36105

Rouzier, R., Rajan, R., Wagner, P., Hess, K. R., Gold, D. L., Stec, J., et al. (2005). Microtubule-associated protein tau: a marker of paclitaxel sensitivity in breast cancer. Proc. Natl. Acad. Sci. U S A 102, 8315-8320. doi: 10.1073/pnas. 0408974102

Sahara, N., Tomiyama, T., and Mori, H. (2000). Missense point mutations of tau to segregate with FTDP-17 exhibit site-specific effects on microtubule structure in COS cells: a novel action of R406W mutation. J. Neurosci. Res. 60, 380-387. doi: 10.1002/(sici)1097-4547(20000501)60:3<380::aid-jnr13>3.0.co;2-5

Santarella, R. A., Skiniotis, G., Goldie, K. N., Tittmann, P., Gross, H., Mandelkow, E.-M., et al. (2004). Surface-decoration of microtubules by human tau. J. Mol. Biol. 339, 539-553. doi: 10.1016/j.jmb.2004.04.008

Schneider, A., Biernat, J., von Bergen, M., Mandelkow, E., and Mandelkow, E. M. (1999). Phosphorylation that detaches tau protein from microtubules (Ser262, Ser214) also protects it against aggregation into Alzheimer paired helical filaments. Biochemistry 38, 3549-3558. doi: 10.1021/bi981874p

Schwalbe, M., Kadavath, H., Biernat, J., Ozenne, V., Blackledge, M., Mandelkow, E., et al. (2015). Structural impact of tau phosphorylation at threonine 231. Structure 23, 1448-1458. doi: 10.1016/j.str.2015. 06.002

Schwalbe, M., Ozenne, V., Bibow, S., Jaremko, M., Jaremko, L., Gajda, M., et al. (2014). Predictive atomic resolution descriptions of intrinsically disordered hTau40 and $\alpha$-synuclein in solution from NMR and small angle scattering. Structure 22, 238-249. doi: 10.1016/j.str.2013.10.020

Scott, C. W., Blowers, D. P., Barth, P. T., Lo, M. M., Salama, A. I., and Caputo, C. B. (1991). Differences in the abilities of human tau isoforms to promote microtubule assembly. J. Neurosci. Res. 30, 154-162. doi: 10.1002/jnr. 490300116

Scott, C. W., Spreen, R. C., Herman, J. L., Chow, F. P., Davison, M. D., Young, J., et al. (1993). Phosphorylation of recombinant tau by cAMP-dependent protein kinase. Identification of phosphorylation sites and effect on microtubule assembly. J. Biol. Chem. 268, 1166-1173.

Selenica, M.-L., Benner, L., Housley, S. B., Manchec, B., Lee, D. C., Nash, K. R., et al. (2014). Histone deacetylase 6 inhibition improves memory and reduces total tau levels in a mouse model of tau deposition. Alzheimers Res. Ther. 6:12. doi: $10.1186 /$ alzrt241 
Sengupta, A., Kabat, J., Novak, M., Wu, Q., Grundke-Iqbal, I., and Iqbal, K. (1998). Phosphorylation of tau at both Thr 231 and Ser 262 is required for maximal inhibition of its binding to microtubules. Arch. Biochem. Biophys. 357, 299-309. doi: 10.1006/abbi.1998.0813

Shashi, V., Magiera, M. M., Klein, D., Zaki, M., Schoch, K., Rudnik-Schöneborn, S., et al. (2018). Loss of tubulin deglutamylase CCP1 causes infantileonset neurodegeneration. EMBO J. 37:e100540. doi: 10.15252/embj.2018 100540

Sibille, N., Huvent, I., Fauquant, C., Verdegem, D., Amniai, L., Leroy, A., et al. (2012). Structural characterization by nuclear magnetic resonance of the impact of phosphorylation in the proline-rich region of the disordered Tau protein. Proteins 80, 454-462. doi: 10.1002/prot.23210

Sillen, A., Barbier, P., Landrieu, I., Lefebvre, S., Wieruszeski, J. M., Leroy, A., et al. (2007). NMR investigation of the interaction between the neuronal protein tau and the microtubules. Biochemistry 46, 3055-3064. doi: 10.1021/bi06 $1920 \mathrm{i}$

Smoter, M., Bodnar, L., Grala, B., Stec, R., Zieniuk, K., Kozlowski, W., et al. (2013). Tau protein as a potential predictive marker in epithelial ovarian cancer patients treated with paclitaxel/platinum first-line chemotherapy. J. Exp. Clin. Cancer Res. 32:25. doi: 10.1186/1756-9966-32-25

Szyk, A., Deaconescu, A. M., Spector, J., Goodman, B., Valenstein, M. L., Ziolkowska, N. E., et al. (2014). Molecular basis for age-dependent microtubule acetylation by tubulin acetyltransferase. Cell 157, 1405-1415. doi: 10.1016/j.cell. 2014.03.061

Tompa, P., and Fuxreiter, M. (2008). Fuzzy complexes: polymorphism and structural disorder in protein-protein interactions. Trends Biochem. Sci. 33, 2-8. doi: 10.1016/j.tibs.2007.10.003

Trinczek, B., Biernat, J., Baumann, K., Mandelkow, E. M., and Mandelkow, E. (1995). Domains of tau protein, differential phosphorylation and dynamic instability of microtubules. Mol. Biol. Cell 6, 1887-1902. doi: 10.1091/mbc.6. 12.1887

Tsvetkov, P. O., La Rocca, R., Malesinski, S., and Devred, F. (2019). Characterization of microtubule-associated proteins (MAPs) and tubulin interactions by isothermal titration calorimetry (ITC). Methods Mol. Biol. 1964, 151-165. doi: 10.1007/978-1-4939-9179-2_12

Tugaeva, K. V., Tsvetkov, P. O., and Sluchanko, N. N. (2017). Bacterial co-expression of human Tau protein with protein kinase A and 14-3-3 for studies of 14-3-3/phospho-Tau interaction. PLoS One 12:e178933. doi: 10.1371/journal.pone.0178933

Umahara, T., Uchihara, T., Tsuchiya, K., Nakamura, A., Iwamoto, T., Ikeda, K., et al. (2004). 14-3-3 proteins and $\zeta$ isoform containing neurofibrillary tangles in patients with Alzheimer's disease. Acta Neuropathol. 108, 279-286. doi: 10.1007/s00401-004-0885-4 van der Vaart, B., Akhmanova, A., and Straube, A. (2009). Regulation of microtubule dynamic instability. Biochem. Soc. Trans. 37, 1007-1013. doi: 10.1042/BST0371007

Wegmann, S., Eftekharzadeh, B., Tepper, K., Zoltowska, K. M., Bennett, R. E., Dujardin, S., et al. (2018). Tau protein liquid-liquid phase separation can initiate tau aggregation. EMBO J. 37:e98049. doi: 10.15252/embj.201 798049

Weingarten, M. D., Lockwood, A. H., Hwo, S. Y., and Kirschner, M. W. (1975). A protein factor essential for microtubule assembly. Proc. Natl. Acad. Sci. U S A 72, 1858-1862. doi: 10.1073/pnas.72.5.1858

Wilhelmsen, K. C., Lynch, T., Pavlou, E., Higgins, M., and Nygaard, T. G. (1994). Localization of disinhibition-dementia-parkinsonism-amyotrophy complex to 17q21-22. Am. J. Hum. Genet. 55, 1159-1165.

Witman, G. B., Cleveland, D. W., Weingarten, M. D., and Kirschner, M. W. (1976). Tubulin requires tau for growth onto microtubule initiating sites. Proc. Natl. Acad. Sci. U S A 73, 4070-4074. doi: 10.1073/pnas.73.11.4070

Xiong, Y., Zhao, K., Wu, J., Xu, Z., Jin, S., and Zhang, Y. Q. (2013). HDAC6 mutations rescue human tau-induced microtubule defects in Drosophila. Proc. Natl. Acad. Sci. U S A 110, 4604-4609. doi: 10.1073/pnas. 1207586110

Zhang, R., Alushin, G. M., Brown, A., and Nogales, E. (2015). Mechanistic origin of microtubule dynamic instability and its modulation by EB proteins. Cell 162, 849-859. doi: 10.1016/j.cell.2015.07.012

Zhang, B., Maiti, A., Shively, S., Lakhani, F., McDonald-Jones, G., Bruce, J., et al. (2005). Microtubule-binding drugs offset tau sequestration by stabilizing microtubules and reversing fast axonal transport deficits in a tauopathy model. Proc. Natl. Acad. Sci. U S A 102, 227-231. doi: 10.1073/pnas.04063 61102

Zhang, F., Su, B., Wang, C., Siedlak, S. L., Mondragon-Rodriguez, S., Lee, H.-G., et al. (2015). Posttranslational modifications of $\alpha$-tubulin in Alzheimer disease. Transl. Neurodegener. 4:9. doi: 10.1186/s40035-015-0030-4

Conflict of Interest Statement: The authors declare that the research was conducted in the absence of any commercial or financial relationships that could be construed as a potential conflict of interest.

Copyright (C) 2019 Barbier, Zejneli, Martinho, Lasorsa, Belle, Smet-Nocca, Tsvetkov, Devred and Landrieu. This is an open-access article distributed under the terms of the Creative Commons Attribution License (CC BY). The use, distribution or reproduction in other forums is permitted, provided the original author(s) and the copyright owner(s) are credited and that the original publication in this journal is cited, in accordance with accepted academic practice. No use, distribution or reproduction is permitted which does not comply with these terms. 